\title{
Estudo controlado e randomizado entre o uso de ventilação por pressão positiva intermitente e pressão positiva contínua em vias aéreas em recém-nascidos pré-termo após a extubação traqueal
}

Dissertação apresentada à Faculdade de Medicina da Universidade de São Paulo para obtenção de título de Mestre em Ciências

Área de concentração: Pediatria

Orientadora: Profa. Dra. Edna Maria de Albuquerque Diniz

São Paulo

2007 
Essa Dissertação de Mestrado é dedicada

Aos meus pais,

Osmar Umberto Rizzo

Maria Helena Franco Rizzo

Ao meu marido

William Ricardo Komatsu

E ao meu filho

Felipe Rizzo Komatsu 
Aos meus pais Osmar e Maria Helena

por toda minha formação pessoal, e por me ensinarem a viver com dignidade, carinho e dedicação.

A William, meu marido, pelo exemplo de seriedade, entusiasmo e respeito com a profissão.

A Felipe, meu filho, razão principal de minha busca por um mundo melhor.

Às minhas irmãs Fernanda e Renata, presenças constantes nos momentos de tristeza e alegria, sempre me apoiaram com carinho e torceram pela minha vitória. 
AGRADECIMENTOS 
À Profa. Dra. Edna Maria de Albuquerque Diniz pela oportunidade em realizar este trabalho, por todo ensinamento, paciência, confiança, estímulo e apoio, e pelo exemplo de mestre, médica, pesquisadora e ser humano.

Ao Prof. Flávio Adolfo Costa vaz pela oportunidade e apoio na realização deste trabalho.

Aos médicos da UCINE

Profa. Dra. Maria Esther Jurfest Rivero Ceccon

Profa. Dra. Vera Lúcia Jornada Krebs

Prof. Dr. Rubens Feferbaum

Dra. Renata Amato Vieira

Dra. Meire Nagaiassu

Dra. Marcília Sierro Grassi

Dra. Cristina Erico Yoshimoto pelos ensinamentos, carinho e incentivo durante a minha permanência nesse Serviço.

À Dra. Silvia Espiridião pela oportunidade em realizar este trabalho, tornando possível a sua realização no Hospital Estadual de Santo André.

À Dra. Sandra Frota Gianelo Ávilla pela oportunidade em realizar este trabalho

À Lúcia Cândida S. de Paula pela amizade, incentivo e auxílio durante a coleta dos dados deste trabalho.

À equipe Médica, e principalmente aos Médicos Residentes de Pediatria das instituições participantes do estudo pelo auxílio na coleta dos dados.

Às equipes de Enfermagem e Fisioterapia da UCINE e da UTIN do Hospital Estadual de Santo André que colaboraram de maneira direta ou indireta na realização deste estudo.

Aos Drs. Alexandre Ferraro e Crésio Romeu Pereira pelos ensinamentos e auxílio na análise estatística dos dados.

À Solange, Adriana e demais funcionárias da pós-graduação pela paciência e atenção durante a realização deste trabalho. 
À Nivaldo L. Rocha e Milene R. Rocha pela preocupação e profissionalismo que foram fundamentais em todo o período de minha permanência nesta instituição.

À Mariza Kazue Umetsu

pela cortesia e auxílio na localização de artigos e referências bibliográficas.

Às mães e principalmente aos recém-nascidos que tornaram possível e são a razão deste trabalho. 


\section{NORMATIZAÇÃO ADOTADA}

Esta dissertação está de acordo com as seguintes normas, em vigor no momento desta publicação:

Referências: adaptado do International Committee of Medical Journals Editors (Vancouver).

Universidade de São Paulo. Faculdade de Medicina. Serviço de Biblioteca e Documentação. Guia de apresentação de dissertações, teses e monografias. Elaborado por Anneliese Carneiro da Cunha, Maria Julia de A. L. Freddi, Maria F. Crestana, Marinalva de Souza Aragão, Suely Campos Cardoso, Valéria Vilhena. 2e ed. São Paulo: Serviço de Biblioteca e Documentação, 2005.

Abreviaturas dos títulos dos periódicos de acordo com List of Journals Indexed in Index Medicus. 
Lista de Abreviaturas

Lista de símbolos

Lista de tabelas

Lista de gráficos

Resumo

Summary

1. INTRODUÇÃO

2. OBJETIVOS

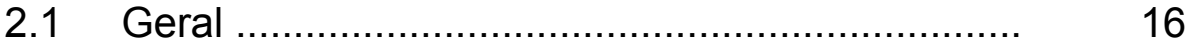

$2.2 \quad$ Específicos ................................................ 16

3. CASUÍSTICA E MÉTODOS ....................................... 17

3.1 Local do estudo ....................................... 18

3.2 Critérios de inclusão .................................... 18

3.3 Critérios de exclusão ..................................... 19

3.4 Tipo de estudo ....................................... 19

3.5 Variáveis estudadas ..................................... 19

3.5.1 Insuficiência respiratória ...................... 19

3.5.2 Falência da extubação ........................ 20

3.5.3 Complicações neurológicas ................. 20

3.5.4 Complicações gastrointestinais ............ 21

3.5.5 Outras variáveis ................................ 21 
3.5.5.1 Obstétrica ................................. 21

3.5.5.2 Recém-nascidos.......................... 21

3.5.5.3 Evolução clínica .......................... 22

3.5.5.4 Complicações da prematuridade.. 23

3.6 Delineamento do estudo .............................. 23

3.7 Fluxograma do estudo ................................. 27

3.8 Análise estatística ....................................... 28

3.9 Protocolo ................................................... 29

3.10 Aprovação do estudo .................................... 29

4. RESULTADOS ….................................................

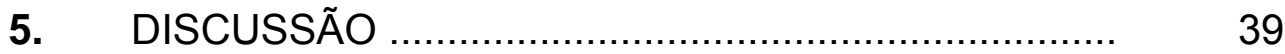

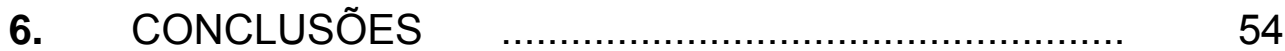

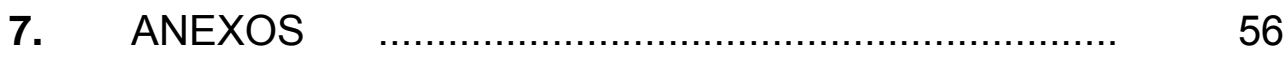

8. REFERÊNCIAS BIBLIOGRÁFICAS .......................... 67 


\begin{tabular}{|c|c|}
\hline AIG & adequado para idade gestacional \\
\hline DBP & displasia broncopulmonar \\
\hline DP & desvio padrão \\
\hline et al. & e outros \\
\hline EBP & extremo baixo peso \\
\hline $\mathrm{FC}$ & freqüência cardíaca \\
\hline $\mathrm{FiO}_{2}$ & fração inspirada de oxigênio \\
\hline FR & freqüência respiratória \\
\hline GIG & grande para idade gestacional \\
\hline $\mathrm{HIC}$ & hemorragia intracraniana \\
\hline IC & intervalo de confiança \\
\hline IG & idade gestacional \\
\hline IR & insuficiência respiratória \\
\hline Ipm & incursões por minuto \\
\hline MAP & pressão média de vias aéreas \\
\hline MBP & muito baixo peso \\
\hline nCPAP & nasal continuous positive airway pressure \\
\hline nIPPV & nasal intermittent positive pressure ventilation \\
\hline $\mathrm{PaO}_{2}$ & pressão arterial de oxigênio \\
\hline $\mathrm{PaCO}_{2}$ & pressão arterial de gás carbônico \\
\hline PCA & persistência do canal arterial \\
\hline
\end{tabular}




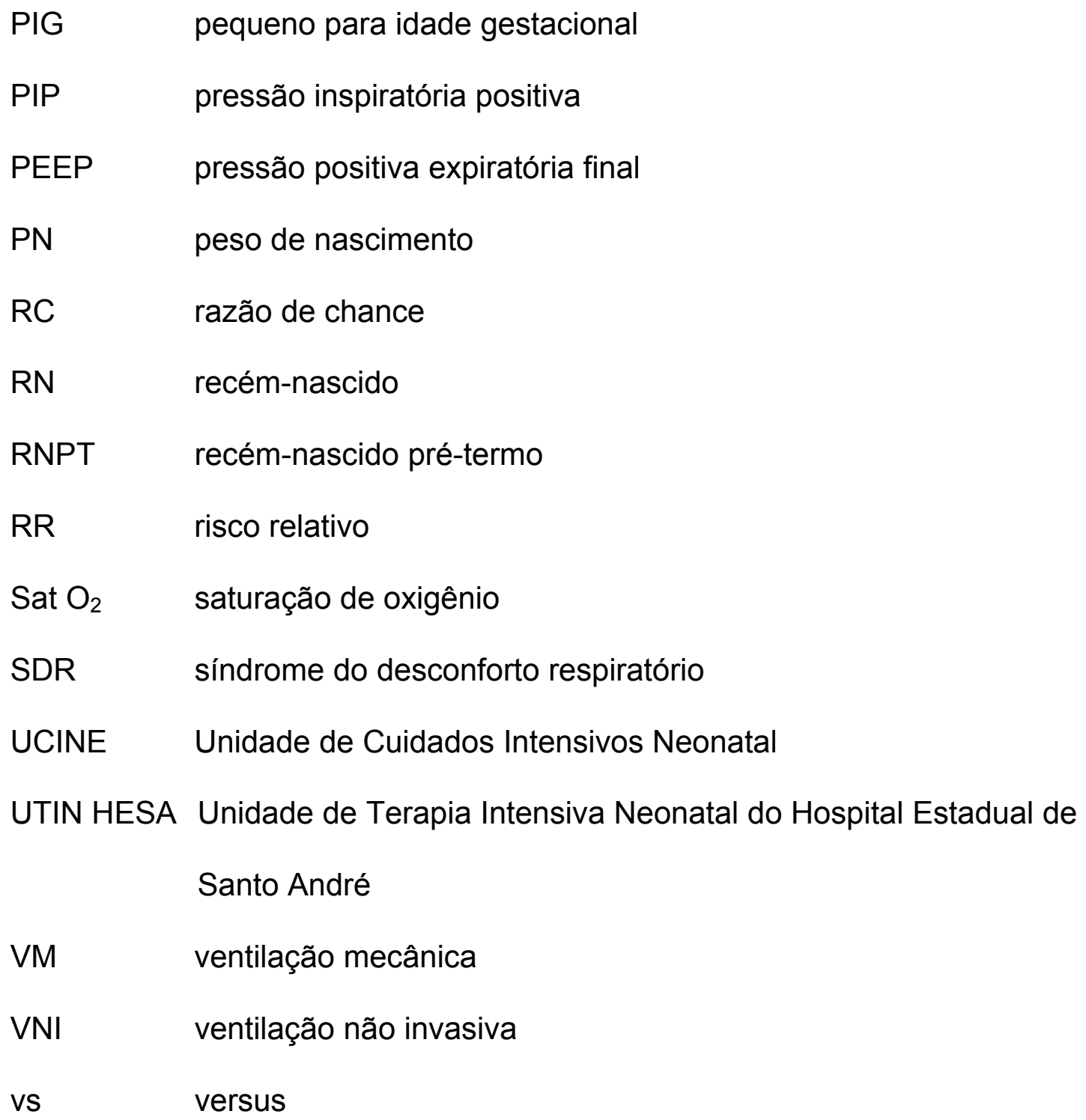




\begin{tabular}{ll}
\hline \hline & \\
$\mathrm{cm} \mathrm{H}_{2} \mathrm{O}$ & centímetro de água \\
$\mathrm{Cm}_{3}$ & centímetro cúbico \\
$\mathrm{dL}$ & decilitro \\
$\mathrm{g}$ & grama \\
$\mathrm{kg}$ & kilograma \\
$\mathrm{L}$ & litro \\
$\mathrm{mg}$ & miligrama \\
$\mathrm{mL}$ & mililitro \\
$\mathrm{mmHg}$ & milímetros de mercúrio \\
$\geq$ & maior ou igual \\
$\leq$ & menor ou igual
\end{tabular}


Tabela 1 - Dados demográficos e clínicos iniciais de 72 RN portadores de insuficiência respiratória submetidos a ventilação por nCPAP ou nIPPV.

Tabela 2 - Medidas terapêuticas e evolução respiratória préextubação de 72 RN submetidos a ventilação por nCPAP ou nIPPV.

Tabela 3 - Tempo de suporte respiratório e incidência de complicações após a extubação em 72 RN submetidos a ventilação por nCPAP ou nIPPV.

Tabela 4 - Peso de nascimento, idade gestacional, classificação e tempo de ventilação mecânica de 17 RNPT submetidos a nIPPV ou nCPAP que apresentaram falha da extubação.

Tabela 5 - Análise univariada, bruta e ajustada para peso de nascimento, idade gestacional, classificação e tempo de ventilação mecânica, do efeito da modalidade nIPPV sobre nCPAP na falha da extubação.

Tabela 6 - Análise multivariada, bruta e com ajuste de possíveis confundidores, do efeito das modalidades de ventilação não invasiva sobre a falha de extubação..

Tabela 7 - Total de casos e incidência de falha de extubação de acordo com o Hospital de origem

Tabela 8 - Resultados da Ultrassonografia de Crânio e de Abdome após a extubação de 72 RN submetidos à ventilação por nCPAP ou nIPPV. 
LISTA DE GRÁFICOS

Gráfico 1 - Principais diagnósticos prévios à extubação de 72 RN portadores de insuficiência respiratória submetidos a ventilação por nCPAP ou nIPPV

Gráfico 2 - Falha da extubação entre os 72 RN portadores de insuficiência respiratória submetidos a ventilação por nCPAP ou nIPPV

Gráfico 3 - $\quad$ Principais causas de falha da extubação nos 17 RNPT 
Komatsu DFR. Estudo controlado e randomizado entre o uso de ventilação por pressão positiva intermitente e pressão positiva contínua em vias aéreas em recém-nascidos pré-termo após a extubação traqueal. São Paulo, 2007. 74p. Dissertação (Mestrado) Faculdade de Medicina, Universidade de São Paulo.

Objetivos: Analisar a frequência de falha da extubação em recém-nascidos pré-termo em ventilação mecânica convencional após a extubação traqueal nos grupos submetidos a ventilação por pressão positiva intermitente por via nasal (nIPPV) e pressão positiva contínua em vias aéreas (nCPAP). Métodos: Foram estudados 72 recém-nascidos pré-termo portadores de insuficiência respiratória, com idade gestacional $<36$ semanas e peso de nascimento $>750$ gramas, que necessitaram de entubação traqueal e ventilação mecânica. Após terem preenchido os critérios de extubação, os recém-nascidos foram submetidos a nIPPV $(n=36)$ ou nCPAP $(n=36)$ de acordo com o resultado da randomização. Os parâmetros iniciais para os RN randomizados para nIPPV foram: $\mathrm{FR}=12$ ipm; $\mathrm{PIP}=16 \mathrm{~cm} \mathrm{H} \mathrm{H}_{2} 0 ; \mathrm{PEEP}=6$ $\mathrm{cm} \mathrm{H} \mathrm{H}_{2} \mathrm{O} ; \mathrm{FiO}_{2} \leq 40 \%$, e para aqueles randomizados para nCPAP foram $\mathrm{P}=6$ $\mathrm{cm} \mathrm{H} \mathrm{H}_{2} \mathrm{O} ; \mathrm{FiO}_{2} \leq 40 \%$. O estudo foi finalizado após um tempo de extubação de 72 horas ou quando o RN preenchesse os critérios de falha na extubação. O projeto de pesquisa foi aprovado pela Comissão de Ética de ambos os Serviços. Resultados: A grande maioria dos RN recebeu pelo menos uma dose de surfactante exógeno (80,5\% no grupo nIPPV e $83,3 \%$ no grupo nCPAP, $P=1)$. $O$ tempo médio de permanência em ventilação mecânica também foi semelhante $(6,2$ e 7,3 dias, $P=0,59$, respectivamente). Entre os $36 \mathrm{RN}$ randomizados para nIPPV, 6 (16,6\%) apresentaram falha de extubação em comparação a $11(30,5 \%)$ dos 36 RN randomizados para nCPAP. Apesar de se ter observado redução relativa de risco de falha de extubação da ordem de 45\% (IC 95\%: 0,23 a 1,32) a favor da nIPPV, esta diferença não alcançou significância estatística. Constatamos uma chance de falha de extubação de 4,38 vezes no grupo nCPAP quando comparado ao grupo nIPPV após excluírmos os RN com peso de nascimento inferior a 1000 gramas, sendo esta diferença estatisticamente significativa ( $P=$ 0,045).Complicações gastrointestinais e neurológicas, além de outras complicações, não ocorreram nos recém nascidos pré-termo submetidos à nIPPV ou nCPAP após a extubação. Conclusões: Embora a distribuição de frequências de falha da extubação tenha mostrado numericamente um menor índice de falha nos RNPT submetidos a nIPPV em relação àqueles submetidos a nCPAP, não houve diferença estatísticamente significante entre os dois modos de suporte ventilatório após a extubação. Ao excluirmos da análise os RN com peso de nascimento inferior a 1000 gramas 
constatamos um aumento estatisticamente significante na chance de falha no grupo nCPAP.

Descritores: 1. Síndrome do desconforto respiratório do recém-nascido 2. Pressão positiva contínua nas vias aéreas 3 . Ventilação com pressão positiva intermitente 4. Prematuro 5. Ensaios clínicos controlados e aleatórios 
Komatsu,DFR. Randomized controlled trial comparing nasal intermittent positive pressure ventilation and nasal continuous positive airway pressure in premature infants after tracheal extubation. São Paulo, 2007. 74p. Dissertation - Faculdade de Medicina, Universidade de São Paulo.

Objetive: To analyze the frequency of failed extubation in premature infants following mechanical ventilation placed on either nasal intermittent positive pressure ventilation (nIPPV) or nasal continuous positive airway pressure (nCPAP) after endotracheal tube removal. Methods: Seventy two premature infants with respiratory insufficiency and gestacional age $<36$ weeks and birthweight $>750$ grams, who needed endotracheal intubation and mechanical ventilation were studied. Once the babies reached the criteria for exubation, they were randomized to either nIPPV $(n=36)$ or to nCPAP $(n=36)$ in accordance with the randomization resulted. The inicial settings for those randomized to nIPPV were: rate=12; $\mathrm{PIP}=16 \mathrm{~cm} \mathrm{H} \mathrm{H}_{2} 0 ; \mathrm{PEEP}=6 \mathrm{~cm} \mathrm{H} \mathrm{H}_{2} 0 ; \mathrm{FiO}_{2}$ $\leq 40 \%$, and for those randomized to nCPAP were $\mathrm{P}=6 \mathrm{~cm} \mathrm{H} \mathrm{H}_{2} \mathrm{O} ; \mathrm{FiO}_{2} \leq 40 \%$. The study ended after 72 hours or when the infants reached the criteria of failure of the extubation. Ethical Approval was obtained from the Hospital. Results: Most received at least one dose of exogenous surfactant $(80,5 \%$ in the nIPPV group and $83,3 \%$ in the nCPAP group, $\mathrm{P}=1$ ). The average time of mechanical ventilation also was similar $(6.2$ and 7.3 days respectively, $P=$ 0.59). Of the 36 infants randomized to nIPPV, six $(16,6 \%)$ failed extubation in comparison to $11(30,5 \%)$ of the 36 newborn randomized to nCPAP. Although to have itself observed relative reduction of risk failure of extubation the order of $45 \%(95 \% \mathrm{Cl}: 0,23$ to 1,32$)$ in the group nIPPV, this difference did not reach significance statistics. We verified a increaset of failure extubation of 4,38 times in the nCPAP group when compared to the group nIPPV after excluded premature infant from birthweight less than 1000 grams, this difference was significant $(P=0,045)$. Gastrointestinal and neurological complications, beyond other complications, had no occurred in premature infants submitted to nIPPV or nCPAP after the extubation. Conclusions: Although the distribution of frequencies has numerically shown a lesser index of failure in premature infants submitted to nIPPV in relation to those submitted to nCPAP after extubation they did not have statistical significance difference between the two ways of ventilatory support. To the excluded of the analysis premature infant with birthweight less than 1000 grams, verified an increase significant in the failure extubation in the nCPAP.group . 
Descriptors: 1. Respiratory distress syndrome newborn 2. Continuous positive airway pressure 3 . Intermittent positive-pressure ventilation 4 . Infant premature 5. Randomized controlled trials 
1 - INTRODUÇÃO 
Diante dos avanços da neonatologia, particularmente na assistência ventilatória, cada vez mais recém-nascidos (RN) prematuros têm sobrevivido, especialmente aqueles de muito baixo peso (MBP) (Barrington et al., 200; Khalaf et al., 2001; Tomminska et al., 2007).

Desconforto respiratório é uma das patologias mais comuns que acometem esses RN, com uma incidência que varia de 3 a $7 \%$ de todos os nascimentos. Estudos de autópsia revelaram que 32 a $52 \%$ de todos os óbitos perinatais são devido a patologias respiratórias, as quais na maioria das vezes necessitam de suporte respiratório através do uso de ventilação mecânica (Malik et al.,2003).

Os principais objetivos deste tipo de intervenção no período neonatal são: minimizar as alterações da relação ventilação/perfusão melhorando assim as trocas gasosas e mantendo níveis adequados da $\mathrm{PaO}_{2}$ (pressão arterial de oxigênio), otimizar a ventilação alveolar mantendo a $\mathrm{PaCO}_{2}$ (pressão arterial de gás carbônico) dentro da faixa de normalidade, reexpandir áreas atelectásicas e diminuir o trabalho respiratório evitando desta forma a fadiga muscular (Guinsburg e Miyoshi, 1998).

A conduta do ponto de vista respiratório desses $\mathrm{RN}$ tem sido direcionada a minimizar a necessidade de ventilação mecânica (VM) prolongada através de uma assistência respiratória pouco agressiva e pelo tempo menor possível para reduzir o barotrauma e a toxicidade pelo oxigênio (Jobe et al., 2001; Bancalari et al,2001; Khalaf et al., 2001). Lembramos que a ventilação de um RN prematuro implica em risco diário e contínuo de lesão pulmonar. 
Entre as causas de insuficiência respiratória no período neonatal destacamos a Síndrome do Desconforto Respiratório (SDR) ou Doença das Membranas Hialinas (DMH), que é uma das patologias respiratórias mais freqüentes no RN pré-termo (RNPT). Caracteriza-se por insuficiência respiratória progressiva que pode se iniciar ao nascimento e atingir maior gravidade em torno de 48 horas de vida caso não sejam tomadas medidas terapêuticas adequadas. Sua etiologia primária é a deficiência de surfactante em vista da imaturidade anatômica e bioquímica pulmonar, a qual guarda relação com a idade gestacional (IG). (Diniz e Vaz, 2000).

A incidência da SDR é inversamente proporcional à IG e ao peso de nascimento (PN). Ocorre em cerca de 60 a $80 \%$ dos RN menores que 28 semanas, 15 a $30 \%$ daqueles entre 32 e 36 semanas e $5 \%$ naqueles maiores que 37 semanas de IG. (Diniz e Vaz, 2000).

Além da prematuridade alguns fatores predispõem à maior incidência da SDR como: filhos de mães diabéticas, gravidez múltipla, parto cesáreo eletivo, asfixia perinatal e história de filhos prévios afetados.

A aplicação cuidadosa das medidas de suporte geral (manutenção de ambiente térmico neutro e hidratação adequada), assistência respiratória (promoção de suporte respiratório que permita manter oxigenação e ventilação adequadas) e a terapia de reposição com surfactante exógeno, diminuem consequentemente a gravidade da doença.

Com a introdução do surfactante exógeno no tratamento da SDR, tem havido uma melhora adicional na sobrevivência dos RNPT. Sabemos que 
após a instilação do surfactante ocorre uma melhora rápida da oxigenação arterial e da complacência pulmonar. (Ramanathan, 2007). Nas primeiras horas após a sua administração observa-se aumento da capacidade residual funcional, propiciando uma maior superfície para as trocas gasosas, melhorando a relação ventilação/perfusão, diminuindo o "shunt" intrapulmonar e, conseqüentemente, corrigindo a hipoxemia (Bancalari e Del Moral, 2001).

No entanto, apesar do uso do surfactante exógeno, quer na forma profilática ou com a SDR já instalada, ainda constata-se uma morbidade elevada, principalmente naqueles RNPT que necessitam de VM por período prolongado.

Ao mesmo tempo em que a ventilação mecânica é essencial para auxiliar a respiração dos RNPT, a mesma envolve risco elevado de complicações graves como: síndrome de escape de ar, representado principalmente pelo enfisema intersticial pulmonar observado particularmente no RN de MBP; pneumotórax, o qual pode ocorrer em cerca de um terço dos pacientes (Rugolo,1999); e o aumento de pneumonia nosocomial, que é uma das complicações mais freqüentes relacionadas à ventilação mecânica. Além disto a ventilação mecânica constitue um fator de risco importante no desenvolvimento da displasia broncopulmonar (DBP) (Jobe e Bancalari, 2001). Lesão de vias aéreas com necrose tecidual e muitas vezes evolução com edema após a extubação ou estenose subglótica têm sido descritas como complicações graves da VM (Page et al., 1998). 
A DBP constitui uma das mais freqüentes causas de doença pulmonar crônica na infância, ocorrendo em mais de $20 \%$ dos RNPT com SDR e em $50 \%$ dos RNPT com peso inferior a 1250 gramas ao nascimento que sobreviveram além de 28 dias de vida, sendo raramente constatada em RN de termo (Diniz e Vaz, 2000; Jobe e Bancalari, 2001). O desenvolvimento de DBP é significantemente associado com o peso de nascimento e idade gestacional (Edwards et al, 1977; Horbar et al., 1988; Van Lierde et al.,1992). A DBP parece resultar de um processo multifatorial extenso que se inicia com uma lesão pulmonar aguda, causada por vários mecanismos em RN susceptível, principalmente pelo barotrauma (Tooley, 1979). Recentemente a redução do tempo de ventilação pulmonar mecânica tem sido descrita como uma das melhores práticas, visando reduzir a lesão pulmonar e prevenir a DBP em prematuros internados em unidades de cuidados intensivos neonatais (Barrington et al., 2001).

Deste modo, tentativas de extubação o mais precoce possível deverão ser realizadas.

A condição ideal para garantir o desmame bem sucedido da ventilação mecânica ainda é controversa. As dificuldades em descontinuar o suporte ventilatório se devem à inadequada compreensão dos mecanismos responsáveis pela falha da extubação e ausência de parâmetros que sejam suficientemente sensíveis em predizer o sucesso do desmame (Dries, 1997).

Para que o processo de desmame ocorra de forma satisfatória, alguns critérios e condições clínicas básicas devem estar presentes, destacando-se a estabilidade hemodinâmica e cardiovascular, e da mecânica respiratória. 
Além disto deve haver uma adequada função do centro respiratório, melhora ou resolução da causa básica da insuficiência respiratória, ausência de broncoconstrição e de bloqueio neuro-muscular nas 24 horas precedentes à extubação, estando o paciente preferencialmente sem sedação. Quanto aos parâmetros do ventilador não há ainda valores pré-determinados que orientem com segurança o início do desmame. No entanto, considera-se que preenchidas as condições descritas acima e estando o $\mathrm{RN}$ com $\mathrm{FiO}_{2}$ (fração inspirada de oxigênio) menor que 40\%, PEEP (pressão positiva expiratória final) menor que $5 \mathrm{cmH}_{2} \mathrm{O}$, PIP (pressão inspiratória positiva) menor que 15 $\mathrm{cmH}_{2} \mathrm{O}$, o paciente teria indicação de ser desmamado do respirador. Salientamos que a extubação deve ser realizada quando o RN estiver clínica e gasometricamente capaz de manter a ventilação espontânea utilizando parâmetros baixos do respirador (Antunes et al., 2002).

No RN de MBP a falência da extubação constitue um problema freqüente devido principalmente a facilidade maior que esses RN apresentam para hipoventilação e atelectasia, levando-os a crises de apnéia grave (Barrington et al., 2001; Lin et al., 1998).

Sabemos que no RN, particularmente aqueles de muito baixo peso, quanto menor o tempo de VM, e portanto de barotrauma, maiores serão os benefícios, ou seja, haverá menor incidência de lesões traqueais, laríngeas, além de menor chance de infecção hospitalar e sepse, o que aumenta a morbidade e gravidade da DBP (Barrington et al., 2001; Ramanathan, 2007).

De acordo com Parker (1996) a taxa de falência após a extubação tem sido de 17 a $19 \%$ em adultos e de 22 a $28 \%$ em RNPT. Outros autores 
têm relatado falha da extubação que varia de 22 a 33\% em RN prematuros (Khan et al.,1996; Mador, 1998; Kavvadia et al., 2000).

A falha de extubação é preocupante pois além de prolongar o tempo de suporte ventilatório, aumenta também a probabilidade de ocorrência de trauma de vias aéreas e de infecção nosocomial com a re-intubação.

A falha da extubação conforme já salientamos é em grande parte devido à atelectasia alveolar ou lobar associada à retenção de secreções e redução do esforço respiratório (Kavvadia et al., 2000). Estas dificuldades são freqüentes e comumente presentes no RNPT, sendo diretamente proporcionais à idade gestacional.

As metilxantinas têm sido utilizadas para auxiliar na força respiratória, e consequentemente na extubação. O maior benefício tem sido descrito em $\mathrm{RN}$ com peso de nascimento inferior a 1000 gramas $(\mathrm{g})$ durante a primeira semana de vida (Henderson-Smart e Davis, 1998).

Alguns estudos de metanálise analisados por Davis et al. (2001) têm mostrado a utilização de diversas intervenções para reduzir a atelectasia ou falência após a extubação do RNPT. Entre estas intervenções destaca-se o uso de CPAP (continuous posivite airway pressure), que tem a finalidade de manter uma pressão positiva nas vias aéreas durante a respiração espontânea. Pode ser utilizado através de tubo endotraqueal, máscara facial, tubos nasofaríngeos ou nasais, e parece reduzir a freqüência das falhas de extubação do RN (Khalaf et al., 2001; Davis e Henderson-Smart, 2001). Esta é a intervenção que tem sido mais comumente utilizada como modo de suporte ventilatório após a extubação (Jeena et al., 2002). 
Efeitos benéficos do CPAP incluem a prevenção de atelectasia, melhora na oxigenação e diminuição da apnéia (De Klerk et al., 2001).

Miller et al. (1990) sugeriram que a aplicação de nCPAP diminue a resistência supra-glótica diretamente por estimular a mecânica respiratória, sendo este o mecanismo primário para redução da apnéia em RNPT. Os autores demonstraram ainda que a nCPAP melhora o movimento sincrônico tóraco-abdominal sugerindo uma melhora respiratória. Porém ainda tem sido demonstrada falha de extubação em torno de $28 \%$ através desta técnica, o que justifica o desenvolvimento e/ou aprimoramento de métodos para melhorar a eficácia do CPAP (Davis et al., 2001).

Destacamos que a criança em CPAP em geral tem um aumento no esforço respiratório para produzir um adequado volume tidal, se isto não acontecer poderá ocorrer falência respiratória e a re-intubação poderá ser necessária.

Mais recentemente tem sido descrito um novo método de ventilação não invasiva por via nasal, que é a nIPPV (nasal Intermittent Positive Pressure Ventilation) em RN extubados com a finalidade de diminuir a incidência de falha da extubação. Esta modalidade de ventilação parece assegurar um melhor suporte respiratório, particularmente em RN de MBP, diminuindo a necessidade de re-intubação endotraqueal (Barrington et al., 2001; Kiciman et al., 1998). A nIPPV permite fornecer CPAP aos RN no modo de IPPV, isto é, ventilação por pressão positiva intermitente, podendo ser utilizada também de maneira sincronizada, denominada nSIPPV (nasal Syncronized Intermittent Positive Pressure Ventilation) (De Paoli et al., 
2003). Atualmente, não há nenhuma evidência direta para a superioridade do modo sincronizado da nIPPV sobre o modo não sincronizado. O uso do modo sincronizado requer um ventilador capaz de promover esse tipo de ventilação. No entanto, métodos menos dispendiosos de uso de nCPAP existem e podem ser importantes em hospitais com recursos reduzidos (Davis et al., 2001).

Os RNPT freqüentemente apresentam dificuldades na transição da ventilação mecânica via endotraqueal para respiração espontânea em vista da ocorrência de fadiga do diafragma e dos músculos respiratórios que são recrutados na fase de resolução da SDR (Moretti et al., 1999). Desse ponto de vista, a nIPPV pode ser uma alternativa mais atrativa que a nCPAP no processo de desmame já que reduz o esforço respiratório do paciente.

Reforçamos que a respiração por pressão positiva poderá ser realizada apenas quando o RN for capaz de iniciar o esforço respiratório ou quando for capaz de manter a glote aberta, ou ainda após um curto intervalo de apnéia (menos de 20 segundos).

A ventilação não invasiva por máscara nasal ou tubos nasais tem sido praticada em adultos e crianças maiores com registros de eficácia e redução daquelas complicações constatadas com a intubação orotraqueal. Embora haja alguns estudos sobre o uso de nIPPV (seja na modalidade sincronizada ou não) no RN, principalmente após a introdução do surfactante exógeno, em comparação com o nCPAP isoladamente, a maioria das pesquisas que tem avaliado a eficácia da ventilação por nIPPV é constituída por pequeno número de RN, observação por período curto ou 
sem avaliação da função pulmonar (Ryan et al., 1989; Lin et al., 1998; Barrington et al., 2001).

Ryan et al. (1989) realizaram estudo prospectivo randomizado em 20 RN com idade gestacional inferior a 32 semanas, comparando a nIPPV e a nCPAP nas primeiras 6 horas após a extubação quanto a ocorrência de apnéia e/ou bradicardia. Todos os RN receberam aminofilina. Em seu estudo, os autores constataram que a nIPPV não reduziu a incidência de apnéia após a extubação quando comparado com o nCPAP, bem como a relação $\mathrm{PaCO}_{2} / \mathrm{PaO}_{2}$ e diferença alvéolo capilar que não foram significantes. De acordo com os autores o pequeno número de RN estudados e o curto período da pesquisa não permitiram uma conclusão definitiva, sugerindo-se mais estudos a respeito.

Lin et al. (1998) em um estudo prospectivo randomizado avaliaram a eficácia da nIPPV no tratamento da apnéia da prematuridade em uma amostra composta por 34 RNPT com média de IG de 27,6 semanas, dos quais 18 foram randomizados para nIPPV e 16 para nCPAP. Os autores constataram uma redução do número de episódios de apnéia significantemente maior no grupo nIPPV quando comparado ao grupo nCPAP $(P=0,02)$, e concluíram que a nIPPV foi mais efetiva que a nCPAP em reduzir a freqüência de episódios de apnéia durante o período de apenas 4 horas de observação após a extubação.

Kiciman et al. (1998) em sua pesquisa avaliaram a assincronia dos movimentos tóraco-abdominais em 14 RNPT com média de IG de 30 semanas submetidos à CPAP por via traqueal, a nCPAP e a nSIPPV. Os 
autores constataram que a assincronia tóraco-abdominal foi menor nos RNPT submetidos à nCPAP quando comparado ao CPAP traqueal e também foi menor naqueles RN submetidos à nSIPPV quando comparados com a nCPAP. Concluíram que a nSIPPV pode ser uma alternativa de tratamento para RNPT que necessitam de VM, uma vez que a mesma diminuiu a assincronia tóraco-abdominal, por diminuir a resistência pelos tubos nasais e aumentar a estabilidade da parede torácica, resultando em uma melhoria da mecânica pulmonar.

Moretti et al. (1999) realizaram estudo prospectivo randomizado envolvendo 11 RNPT com intubação orotraqueal prévia devido a SDR, destes, seis foram randomizados para nSIPPV e cinco para nCPAP. Os autores observaram que durante a nSIPPV os valores da $\mathrm{PaCO}_{2}$ transcutânea foram significativamente menores que durante a nCPAP $(\mathrm{P}<0,01)$ enquanto que a $\mathrm{PaO}_{2}$ transcutânea foi apenas ligeiramente mais alta, não mostrando diferença estatisticamente significativa. Relataram ainda que durante a nSIPPV, os volumes pulmonares (volume tidal e volume minuto) foram significativamente maiores quando comparados àqueles na nCPAP. Os autores concluíram que a nSIPPV pode ser realizada em RN de MBP sendo capaz de manter uma ventilação efetiva com redução do esforço respiratório. Acreditam que mais estudos são justificáveis para se determinar se esses efeitos da ventilação nasal sincronizada poderiam resultar em uma melhoria clínica no acompanhamento dos RNPT.

Friedlich et al. (1999) avaliaram 41 RN de MBP (média de PN de 963 gramas) por um período de 48 horas após a extubação, dos quais 22 RN 
foram randomizados para receber nSIPPV e 19 para nCPAP. Os autores encontraram menor incidência de falência de extubação quando em nSIPPV do que em nCPAP (5\% vs $37 \%)$.

Em sua metanálise, Davis et al. (2001) não constataram perfuração gastrintestinal em nenhum RN e também não observaram diferença na incidência de doença pulmonar crônica. Os autores concluíram que a nIPPV é um método útil em RNPT e seu uso reduziu a incidência de falência da extubação mais eficientemente que a nCPAP. No entanto, diante do pequeno número de RN randomizados para nIPPV sugeriram a necessidade de mais estudos com número maior de RN para se obter melhores conclusões sobre o uso da nIPPV versus nCPAP e sua eficácia no tratamento da apnéia da prematuridade.

Barrington et al. (2001) em um estudo randomizado avaliaram 54 RN com peso de nascimento inferior a 1251 gramas por um período de 72 horas após a extubação. Observaram menor incidência na falência da extubação nos RN que utilizaram a modalidade nSIPPV em comparação a nCPAP ( $\mathrm{P}<0,05)$, sendo isto atribuído à menor incidência de apnéia e de hipercapnia em ambos os grupos. Os autores não constataram ocorrência de distensão abdominal ou intolerância alimentar, bem como perfuração gastrointestinal e enterocolite necrosante nos grupos de RN.

Khalaf et al. (2001) realizaram estudo prospectivo randomizado controlado em 64 RNPT com idade gestacional inferior a 34 semanas e diagnóstico de síndrome de desconforto respiratório. Os RN foram randomizados para nSIPPV ou nCPAP após extubação da VM. Constataram 
uma taxa de sucesso de $94 \%$ (32/34) para aqueles em nSIPPV e para aqueles em nCPAP uma taxa de 60\% (18/30) com $P=0,01$. O principal motivo de falha da extubação foi a ocorrência de apnéia em ambos os grupos, mesmo assim os autores constataram uma pequena incidência de apnéia (41\%), sendo 11 casos para a nSIPPV e 14 casos para a nCPAP. Os autores não constataram efeitos adversos em ambos os grupos. Concluíram que a nSIPPV é mais eficaz que a nCPAP no desmame de RN com SDR da ventilação mecânica. Concordam ainda que provas de função pulmonar podem ser úteis auxiliando no prognóstico para o sucesso ou não da extubação e recomendam que o nSIPPV seja utilizado como modo primário de extubação, sobretudo em RN com função pulmonar prejudicada.

Tem sido descrito ainda que uma das principais complicações observadas durante o uso da nIPPV é a ocorrência de perfuração gástrica. Garland et al. (1985) em um estudo retrospectivo caso-controle pareado avaliaram 20 RN com perfuração gastrintestinal durante sua evolução. Todos os RN estavam recebendo ventilação com tubos nasais ou máscara facial no momento do diagnóstico, sendo que 11 foram ventilados exclusivamente com tubos nasais ou máscaras faciais. Os autores constataram que os $\mathrm{RN}$ em nSIPPV ou máscara facial tinham maior probabilidade de sofrer perfuração gastrointestinal do que RN ventilados com tubo endotraqueal.

Outras complicações que têm sido descritas durante o uso da ventilação por via nasal são a necrose do septo nasal e a distensão gástrica, com prejuízo da progressão alimentar desses RN. Porém estas 
complicações não foram relatadas ou até mesmo consideradas significantes em nenhum dos estudos anteriores.

Considerando que a reintubação ou a falha da extubação é uma ocorrência comum em prematuros, e diante do pequeno número de estudos relativos ao assunto e falta de consenso entre os diversos métodos sugeridos para tratar estas crianças é que resolvemos realizar uma pesquisa em RNPT extubados após ventilação mecânica com a finalidade de comparar a eficácia da nIPPV em relação a nCPAP na prevenção da reintubação ou falha da extubação desses RNPT.

A nossa hipótese é que os RNPT extubados e colocados em nIPPV apresentem menor incidência de falha de extubação quando comparados àqueles em nCPAP. 
2 - OBJETIVOS 
1. Objetivo Geral

- Analisar a freqüência de falhas de extubação em RNPT portadores de insuficiência respiratória em ventilação mecânica convencional, nas primeiras 72 horas após a extubação nos grupos submetidos à nIPPV ou nCPAP.

2. Objetivos Específicos

- Comparar a eficácia da nIPPV em relação à nCPAP em RNPT, portadores de insuficiência respiratória em ventilação mecânica convencional, após a extubação;

- Analisar a associação entre o peso de nascimento, a idade gestacional, a classificação do RN e o tempo de ventilação mecânica e a freqüência de falha na extubação;

- Descrever a incidência de complicações gastrointestinais (distensão abdominal e perfuração gastrointestinal) e neurológicas durante o período de nIPPV ou nCPAP. 
3 - CASUÍSTICA E MÉTODOS 


\subsection{LOCAL DO ESTUDO}

Este estudo foi realizado na Unidade de Cuidados Intensivos Neonatal (UCINE) do Instituto da Criança do Hospital das Clínicas da Faculdade de Medicina da USP e na Unidade de Terapia Intensiva Neonatal do Hospital Estadual Mário Covas de Santo André (UTIN HESA), ligado à Disciplina de Pediatria da Faculdade de Medicina do ABC. Caracterizam-se por serem unidades que atendem $\mathrm{RN}$ de alto risco, de nível sócio econômico baixo, procedentes principalmente da rede pública de saúde.

A UCINE possui 18 leitos para terapia intensiva neonatal, recebe RN procedentes da maternidade do Hospital das Clínicas da Faculdade de Medicina da USP e de outros hospitais. A UTIN HESA possui 10 leitos para terapia intensiva neonatal, 20 leitos para unidade intermediária e de baixo risco, e possui ainda 20 leitos para alojamento conjunto. Ambos os Serviços contam com ambulatório multidisciplinar de seguimento pediátrico.

\subsection{CRITÉRIOS DE INCLUSÃO}

É constituído por 72 recém-nascidos com idade gestacional menor ou igual a 36 semanas e 6 dias, internados no período de janeiro de 2004 a fevereiro de 2006, portadores de insuficiência respiratória e que necessitaram de entubação orotraqueal e ventilação mecânica. 


\subsection{CRITÉRIOS DE EXCLUSÃO}

Foram excluídos do estudo os RN portadores de:

- anomalias congênitas que comprometessem o sistema cardiorespiratório;

- doenças genéticas;

- doença neuromuscular que comprometesse a ventilação mecânica;

- $\quad$ Apgar inferior a 4 no quinto minuto de vida;

- $\quad$ hemorragia intracraniana graus III e IV;

- malformações do Sistema Nervoso Central;

- $\quad$ depressão do Sistema Nervoso Central por drogas.

\section{$3.4 \quad$ TIPO DE ESTUDO}

Ensaio clínico randomizado

\subsection{VARIÁVEIS ESTUDADAS}

\subsubsection{INSUFICIÊNCIA RESPIRATÓRIA}

Desconforto respiratório caracterizado pela presença de sinais clínicos agudos (tiragem intercostal e/ou subcostal, batimentos de asa de nariz, gemência e cianose) e laboratoriais (necessidade de $\mathrm{FiO}_{2}$ superior a $65 \%$ para manter saturação de $\mathrm{O}_{2}$ entre 90 e $92 \%, \mathrm{PaCO}_{2}$ superior a 65 $\mathrm{mmHg}$ e $\mathrm{pH}<7,25$ ) com necessidade de entubação orotraqueal e ventilação mecânica. 


\subsubsection{FALÊNCIA DA EXTUBAÇÃO}

Ocorrência de sinais clínicos e laboratorias de insuficiência respiratória aguda durante as 72 horas de estudo, que pudessem evoluir para fadiga respiratória e apnéia recorrente grave e necessidade de entubação orotraqueal e ventilação mecânica.

Foi considerada a ocorrência de apnéia a presença de bradicardia (FC < 100 bpm) e/ou queda aguda da saturação observada na oximetria de pulso (Saturação de $\mathrm{O}_{2}<80 \%$ ).

A ocorrência de mais que dois episódios de apnéia em um período de 24 horas necessitando de ventilação com pressão positiva, ou a ocorrência de mais que 6 episódios em 24 horas com duração superior a 20 segundos era considerada apnéia recorrente grave.

\subsubsection{COMPLICAÇÕES NEUROLÓGICAS}

Foi considerada como complicação neurológica a presença de hemorragia peri ou intraventricular de acordo com a classificação de Papile (Papile et al.,1978).

Sempre que possível foi realizada ultrassonografia de crânio previamente ao início do estudo para descartar a ocorrência de hemorragia intracraniana graus III e IV. Na maioria dos RN, a partir de 48 horas após a extubação, foi realizada nova ultrassonografia de crânio com a finalidade de se verificar a ocorrência de hemorragia intracraniana. 


\subsubsection{COMPLICAÇÕES GASTROINTESTINAIS}

Foi considerada como complicação gastrointestinal a ocorrência de intolerância alimentar caracterizada pela presença de resíduo gástrico e/ou distensão abdominal. A maioria dos RN foi submetida a ultrassonografia de abdome a partir de 48 horas após a extubação com o objetivo de se verificar a ocorrência de perfuração gástrica.

Os exames de ultrassonografia foram realizados nos Serviços de Radiologia dos referidos hospitais. Os médicos radiologistas não tinham conhecimento do resultado da randomização.

\subsubsection{OUTRAS VARIÁVEIS}

\subsubsection{OBSTÉTRICA}

- Tempo de ruptura das membranas.

- Tipo de parto: normal ou cesáreo.

\subsubsection{RECÉM-NASCIDOS}

- Idade gestacional: determinada segundo informação materna da data da última menstruação ou através da avaliação clínica, com dados antropométricos, exame físico e neurológico, aplicando-se os métodos de Capurro et al. (1978) ou New Ballard et al. (1991). Nesse caso os RN foram classificados de acordo com a IG em 2 grupos:

$$
\begin{aligned}
& 0<32 \text { semanas; } \\
& 0 \geq 32 \text { semanas. }
\end{aligned}
$$


- Peso de nascimento: conforme o peso de nascimento os RN foram categorizados em 2 grupos:

$$
\begin{aligned}
& \mathrm{o}<1250 \mathrm{~g} \\
& \mathrm{o} \geq 1250 \mathrm{~g} .
\end{aligned}
$$

- Classificação do RN de acordo com o peso de nascimento e a duração da gestação, realizada através das curvas de crescimento fetal de Alexander et al. (1996), em: PIG (pequeno para idade gestacional), AIG (adequado para idade gestacional) e GiG (grande para idade gestacional).

- Sexo: masculino ou feminino.

- Apgar: uma nota inferior a 4 no $5^{\circ}$ minuto de vida foi considerada como asfixia neonatal grave, de acordo com o Consenso da Academia Americana de Pediatria (2002).

\subsubsection{EVOLUÇÃo CLÍNICA}

- Uso de surfactante.

- Uso de aminofilina.

- Idade de extubação.

- Tempo de ventilação mecânica.

- Valor da $\mathrm{FiO}_{2}$ pré-extubação.

- Análise dos gases sanguíneos: Os gases sangüíneos foram monitorizados nas primeiras 24 horas após a extubação e/ou quando fosse necessário. O estudo dos gases foi feito através da coleta de $0,5 \mathrm{ml}$ de sangue por punção arterial, a partir de 6 horas após a extubação, para realização de gasometria. 
- Tempo de uso da modalidade.

- Tempo de suplementação de $\mathrm{O}_{2}$.

\subsubsection{COMPLICAÇÕES DA PREMATURIDADE}

- Sepse: foi diagnosticada pela presença de hemocultura positiva e/ou sinais clínicos e laboratoriais, resultando em decisão clínica de tratamento com antibióticos por pelo menos 7 dias.

- Displasia broncopulmonar: foi definida como dependência de oxigênio com 36 semanas de IG corrigida e/ou presença de alterações parenquimatosas radiográficas características (Jobe e Bancalari, 1991).

- Persistência do canal arterial: caracterizada através do exame clínico e ecocardiograma.

\subsection{DELINEAMENTO DO ESTUDO}

Os RNPT que preencheram os critérios de inclusão do estudo, ou seja que necessitaram de intubação orotraqueal e ventilação mecânica, receberam tratamento para insuficiência respiratória conforme a rotina dos serviços, incluindo o uso de surfactante exógeno. Os RN em ventilação mecânica tiveram os parâmetros ventilatórios reduzidos de acordo com sua evolução clínica e níveis gasométricos. 
Os RN com IG menor que 34 semanas e/ou peso de nascimento inferior a $1500 \mathrm{~g}$ receberam, 12 horas antes da extubação uma dose de ataque de aminofilina $(5 \mathrm{mg} / \mathrm{kg})$ e após, doses de manutenção de 4 $\mathrm{mg} / \mathrm{kg} / \mathrm{dia}$, de 12/12 horas. Nenhum RNPT recebeu tratamento com corticosteróides antes da extubação.

Quando os RN atingiam os critérios de extubação da ventilação mecânica, ou seja, parâmetros ventilatórios: PIP $\leq 16 \mathrm{~cm} \mathrm{H} 20$; PEEP $\leq 4 \mathrm{~cm}$ $\mathrm{H}_{2} \mathrm{O} ; \mathrm{FR}=12$ ipm; $\mathrm{FiO}_{2} \leq 40 \% ; \mathrm{MAP} \leq 7 \mathrm{~cm} \mathrm{H} \mathrm{H}_{2}$; e parâmetros laboratoriais: $\mathrm{pH} \geq 7,25 ; \mathrm{PaO}_{2} \geq 50 \mathrm{mmHg} ; \mathrm{PaCO}_{2} \leq 60 \mathrm{mmHg}$; Hematócrito $\geq 40 \%$ era realizada a extubação seguida da instalação de nIPPV ou nCPAP de acordo com o resultado da randomização. Os $\mathrm{RN}$ eram mantidos no mesmo aparelho de ventilação mecânica, sendo trocado o circuito e colocado o tubo nasal para nCPAP ou nIPPV cujo tamanho era de acordo com o peso do RN.

No estudo foram utilizados ventiladores da marca Newport@ - modelo E100, tanto durante a ventilação mecânica quanto durante a nIPPV ou nCPAP, no caso da UCINE. Foram utilizados tubos nasais da marca Argyle ${ }^{\circledR}$, disponíveis nos tamanhos "extra small" para RN com peso $<1000$ g; "small" para RN com peso entre 1500 e 1000 g; e "large" para RN com peso $>1500 \mathrm{~g}$.

Na UTIN HESA foram utilizados ventiladores da marca Inter III $\circledR$, e tubos nasais da marca Hudson $\mathrm{RCI}$, disponíveis nos tamanhos "zero" para RN com peso < 700 g; "um" para RN com peso entre 700 e 1250 g, "dois" 
para RN com peso entre 1250 e 2000 g, e "três" RN com peso entre 2000 e $3000 \mathrm{~g}$.

A randomização foi realizada no momento da extubação através da utilização de envelopes selados: 36 cartões foram identificados como nIPPV e 36 como nCPAP, colocados dentro de envelopes escuros e distribuídos de maneira aleatória para realização do sorteio, sendo posteriormente desprezados.

Imediatamente após a extubação, os RN recebiam inalação com adrenalina e soro fisiológico e em seguida era instalada a modalidade de ventilação não invasiva sorteada, que era realizada pela própria pesquisadora executante ou pela equipe de fisioterapia na sua ausência.

Os parâmetros iniciais utilizados para os $\mathrm{RN}$ randomizados para nIPPV foram: $F R=12 \mathrm{ipm} ; \mathrm{PIP}=16 \mathrm{~cm} \mathrm{H} 20$; PEEP= $6 \mathrm{~cm} \mathrm{H} \mathrm{H}_{2} \mathrm{O} ; \mathrm{FiO}_{2} \leq$ 40\%. O desmame da nIPPV foi realizado de acordo com a evolução clínica e a análise dos gases sanguíneos, sendo suspenso quando os parâmetros ventilatórios alcançavam os seguintes ajustes: PIP $=14 \mathrm{~cm} \mathrm{H}_{2} 0 ;$ PEEP $<4$ cm $\mathrm{H}_{2} \mathrm{O} ; \mathrm{FiO}_{2}<40 \%$; e $\mathrm{FR}<8$ ipm para manter a saturação de $\mathrm{O}_{2}$ entre 90 e $92 \%$.

Os parâmetros iniciais utilizados para os $\mathrm{RN}$ randomizados para

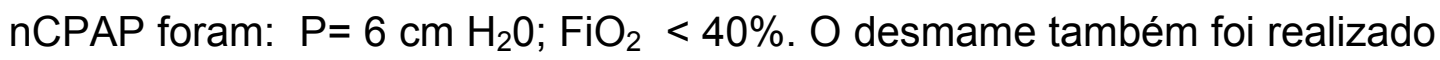
de acordo com a evolução clínica e gasométrica. O nCPAP foi suspenso quando o RN necessitava de uma pressão menor que $4 \mathrm{~cm} \mathrm{H}_{2} \mathrm{O}$ em $\mathrm{FiO}_{2}<$ $40 \%$ para manter a saturação de $\mathrm{O}_{2}$ entre 90 e $92 \%$. 
Os gases sangüíneos foram monitorizados nas primeiras 24 horas após a extubação e/ou quando fosse necessário.

Radiografias, culturas de sangue e ecocardiografia foram feitos quando necessário.

Os RN foram mantidos em monitorização contínua, durante todo o período de estudo e oximetria de pulso, para medir a saturação de hemoglobina com oxigênio.

Durante o estudo os RN eram alimentados por via enteral por meio de sonda orogástrica a cada 3 horas ou por infusão contínua se necessário. 0 resíduo gástrico era medido antes da próxima alimentação, ou a cada 3 horas no caso de dieta por infusão contínua; se o volume do resíduo fosse superior a $20 \%$ do previamente administrado era suspensa a próxima alimentação, particularmente se acompanhado por distensão abdominal.

O estudo foi finalizado após um tempo de extubação de 72 horas ou quando o RN preenchesse os critérios de falha na extubação. O paciente era reavaliado clinicamente e ao menor sinal de insuficiência respiratória eram tomadas as condutas necessárias, como seja: aumento da $\mathrm{FiO}_{2}$; da pressão inspiratória ou expiratória; coleta de gasometria arterial; realização de radiografia de tórax. Após a estabilização do RN os dados clínico, laboratorial e radiológicos foram analisados, e se houvesse necessidade de $\mathrm{FiO}_{2}$ superior a $65 \%$ para manter saturação de $\mathrm{O}_{2}$ entre 90 e $92 \%, \mathrm{PaCO}_{2}$ superior a $60 \mathrm{mmHg}$ e pH< 7,25 e/ou evolução com episódios recorrentes de apnéia, era considerada a falha da extubação sendo realizada a reintubação e re-instalação da ventilação mecânica. 


\subsection{FLUXOGRAMA DO ESTUDO}

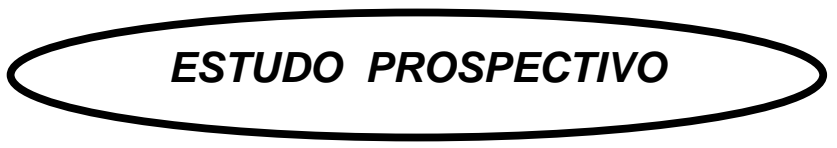
RNPT, IG $\leq 36$ sem6/7, em VM por IR com ou sem tratamento com surfactante exógeno
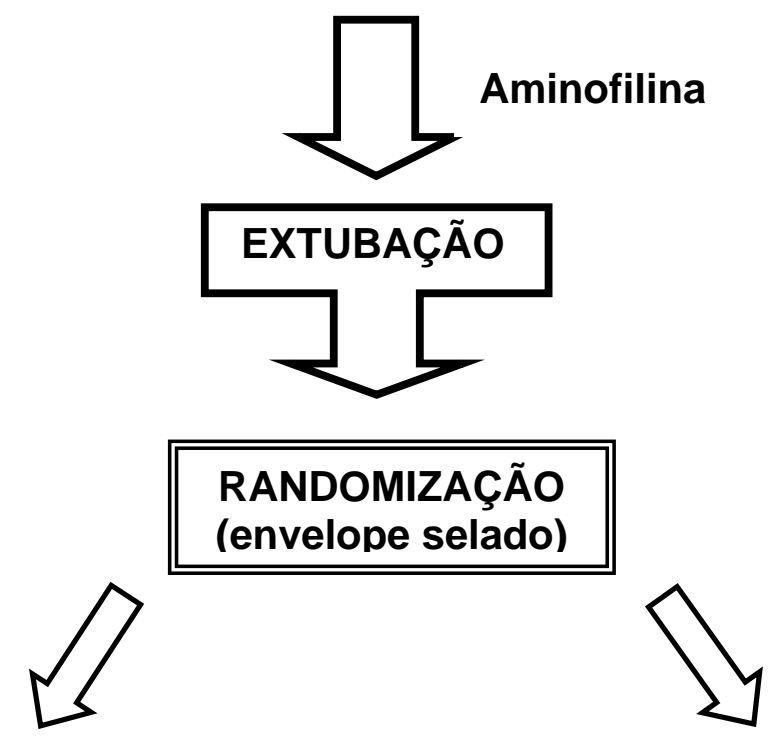
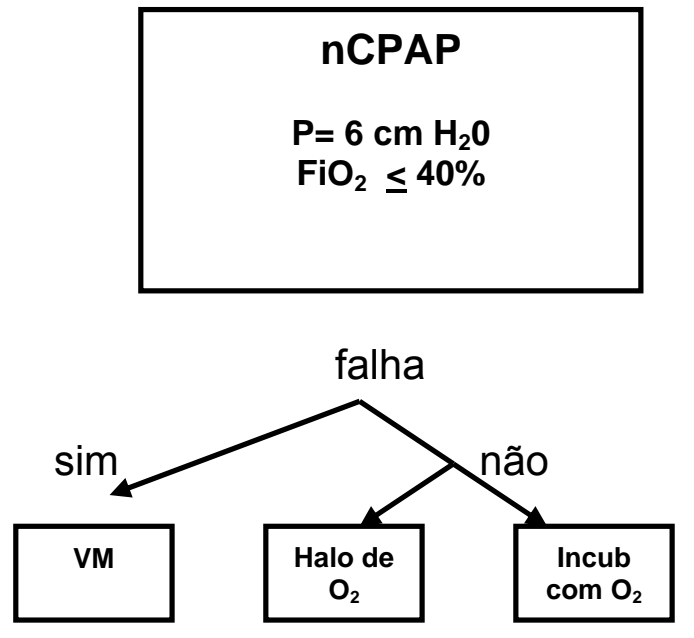
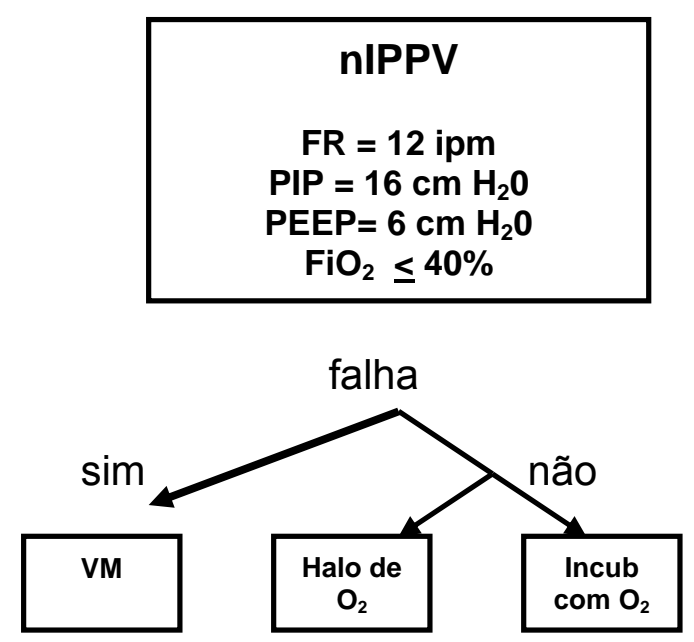

VM: ventilação mecânica; Incub: Incubadora com $\mathrm{O}_{2}$ 


\subsection{ANÁLISE ESTATÍSTICA}

Para as variáveis contínuas foram calculadas as médias e desviospadrão e para as categóricas a freqüência foi apresentada em porcentagens. $\mathrm{Na}$ análise de associação das variáveis independentes com a variável desfecho ("modo de ventilação não invasiva") inicialmente comparou-se as médias segundo o teste "t" de Student e as proporções segundo o quiquadrado. O nível de corte para rejeitar a hipótese de nulidade foi $P \leq 0,05$.

Em um segundo momento realizou-se uma análise univariada com o cálculo dos riscos relativo e seus respectivos intervalos de confiança de $95 \%$, ajustando para potenciais confundidores (tempo de VM, idade gestacional, peso de nascimento e classificação do RN) através do teste de Mantel-Haenszel (efeito ponderado).

Realizou-se ainda uma análise logística multivariada incluindo no modelo todos os potenciais confundidores ao mesmo tempo. O "p de Wald" foi calculado para conhecer a probabilidade da associação "modo de ventilação-falha" ser explicada pelo acaso.

Para a formação e tratamento do banco de dados utilizou-se o programa SOFTWARE Excel®. As análises de associação e univariadas foram feitas no programa EPI-Info® versão 6.0b. A regressão logística multivariada foi feita no programa STATA $8.0 \AA$. 


\section{$3.9 \quad$ PROTOCOLO}

Para cada RN foi preenchido um protocolo específico onde constam: identificação, antecedentes maternos, condições de nascimento e dados do RN: exame clínico à admissão, evolução clínica durante a internação, exames laboratoriais e radiológicos.

\subsection{APROVAÇÃO DO ESTUDO}

O Projeto de pesquisa foi aprovado pela Comissão de Ética de ambos os Serviços.

Consentimento esclarecido foi obtido dos responsáveis para todos os RN. 
4 - RESULTADOS 
Os resultados serão apresentados a seguir, sob a forma de tabelas e gráficos. A análise destes, obedecendo a sistemática de apresentação, será efetuada na próxima parte. 
Tabela 1 - Dados demográficos e clínicos iniciais de 72 RN portadores de insuficiência respiratória submetidos a ventilação por nCPAP ou nIPPV

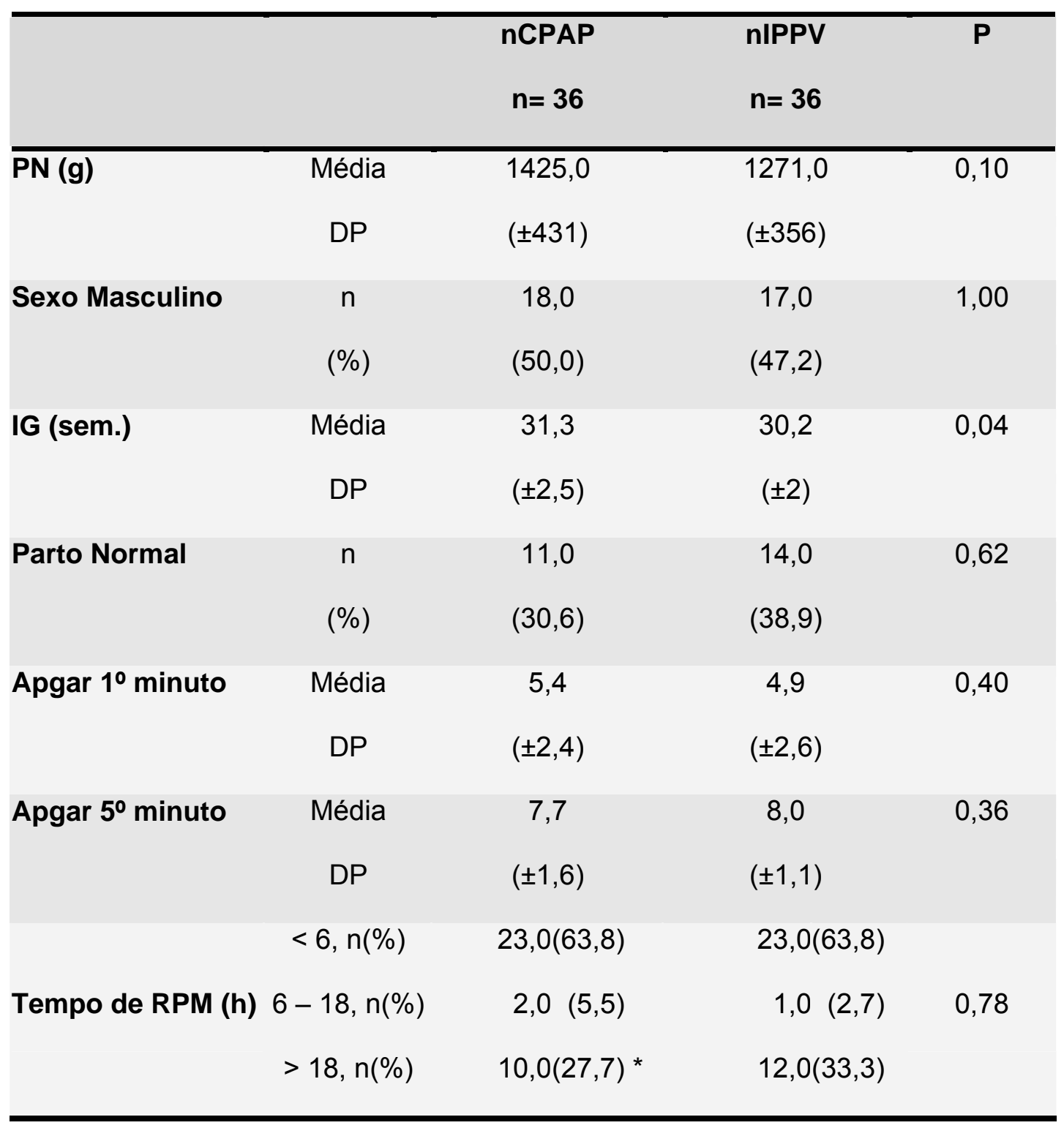

DP: desvio padrão; PN: peso de nascimento; IG: idade gestacional; RPM: rotura prematura das membranas

* Em um RN não havia descrição do tempo de rotura das membranas 
Gráfico 1 - Principais diagnósticos prévios à extubação de 72 RN portadores de insuficiência respiratória submetidos a ventilação por nCPAP ou nIPPV

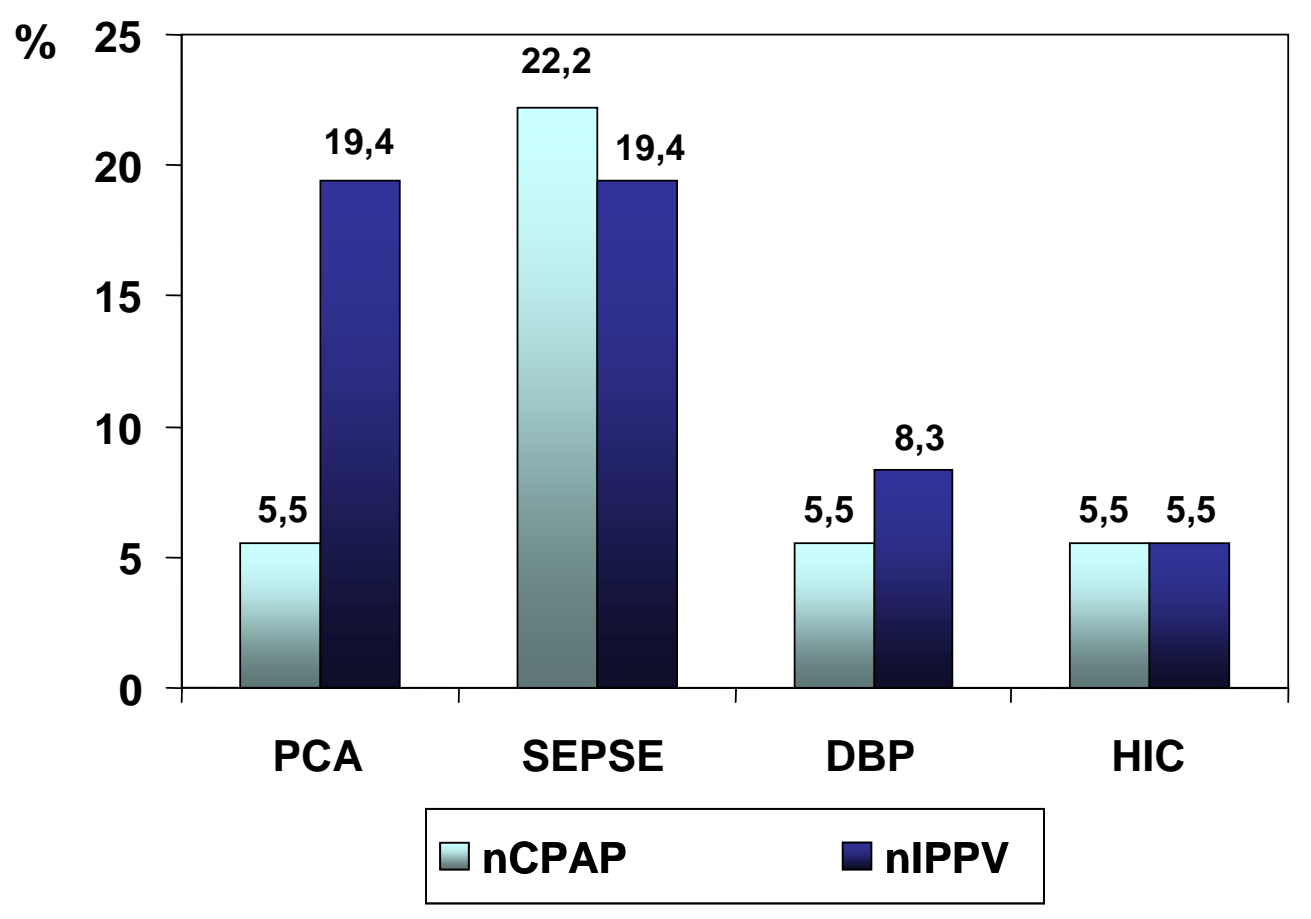

PCA: persistência do canal arterial; DBP: displasia broncopulmonar; HIC: hemorragia intracraniana. 
Tabela 2 - Medidas terapêuticas e evolução respiratória pré-extubação de 72 RN submetidos a ventilação por nCPAP ou nIPPV

\begin{tabular}{ccccc}
\hline & & nCPAP & nIPPV & P \\
& & $\mathrm{n}=\mathbf{3 6}$ & $\mathrm{n}=\mathbf{3 6}$ & \\
\hline Aminofilina & $\mathrm{n}$ & 33,0 & 31,0 & 0,71 \\
Surfactante & $\mathrm{n}$ & 30,0 & 29,0 & \\
exógeno & $(\%)$ & $(83,3)$ & $(80,5)$ & 1,00 \\
Tempo de VM (dias) & Média & 6,2 & 7,3 & 0,59 \\
& DP & $( \pm 8,7)$ & $( \pm 8,7)$ & \\
\hline \multirow{2}{*}{ FiO } & & $(91,6)$ & $(86,1)$ & 0,67 \\
\hline
\end{tabular}

VM: ventilação mecânica.

Tabela 3 - Tempo de suporte respiratório e incidência de complicações após a extubação em 72 RN submetidos a ventilação por nCPAP ou nIPPV

\begin{tabular}{lcccc}
\hline & & nCPAP & nIPPV & P \\
& & $\mathbf{n}=\mathbf{3 6}$ & $\mathbf{n}=\mathbf{3 6}$ & \\
Tempo de uso da VNI (dias) & Média(DP) & $2,0( \pm 2,7)$ & $1,5( \pm 0,7)$ & 0,28 \\
Tempo de $\mathrm{O}_{2}$ pós VNI (dias) & Média(DP) & $3,7(3,7)$ & $3,0(2,2)$ & 0,33 \\
$\mathrm{pH}^{*}$ & Média(DP) & $7,38( \pm 0,07)$ & $7,34( \pm 0,1)$ & 0,05 \\
PaCO $_{2}^{*}$ & Média(DP) & $36,3( \pm 10,7)$ & $34,8( \pm 13,0)$ & 0,59 \\
Resíduo gástrico & $\mathrm{n}(\%)$ & $11(30,5)$ & $12(33,3)$ & 0,80 \\
Distensão abdominal & $\mathrm{n}(\%)$ & $2(5,5)$ & $2(5,5)$ & 1,00 \\
Apnéia & $\mathrm{n}(\%)$ & $6(16,6)$ & $3(8,3)$ & 0,48 \\
\hline
\end{tabular}

VNI: ventilação não invasiva; *: no $1^{\circ}$ dia pós-extubação 
Gráfico 2 - Falha da extubação entre os 72 RN portadores de insuficiência respiratória submetidos a ventilação por nCPAP ou nIPPV

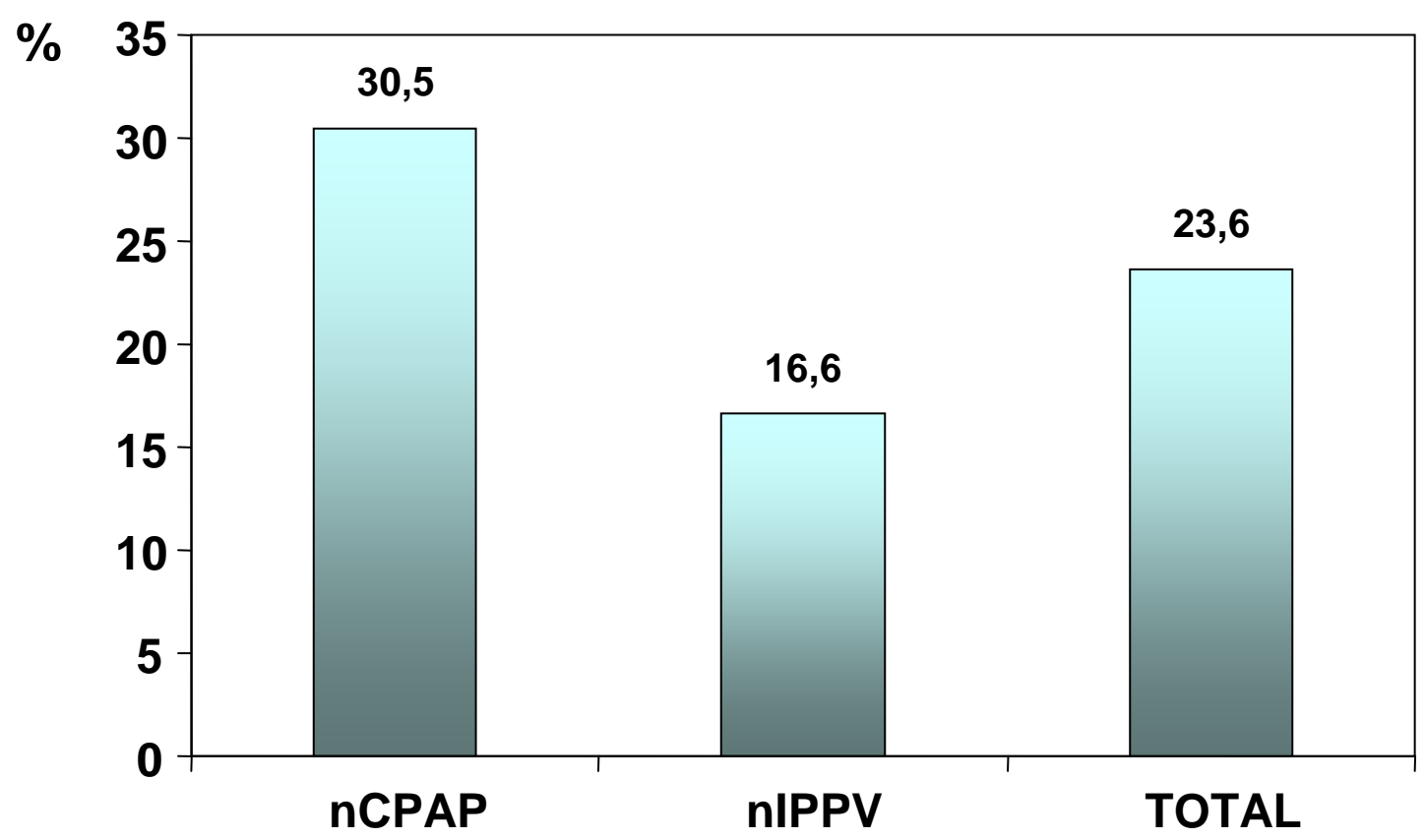


Tabela 4 - Peso de nascimento, idade gestacional, classificação e tempo de ventilação mecânica de 17 RNPT submetidos a nIPPV ou nCPAP que apresentaram falha da extubação

\begin{tabular}{ccccc}
\hline & & nCPAP & nIPPV & GERAL \\
& & f/t (\%) & f/t (\%) & f/t (\%) \\
\hline PN (g) & $<1250$ & $04 / 12(33,3)$ & $05 / 20(25,0)$ & $09 / 32(28,1)$ \\
& $\geq 1250$ & $07 / 24(29,2)$ & $01 / 16(6,1)$ & $08 / 40(20,0)$ \\
\hline IG (semanas) & $<32$ & $06 / 17(35,3)$ & $05 / 27(18,5)$ & $11 / 44(25,0)$ \\
& $\geq 32$ & $05 / 19(26,3)$ & $01 / 09(11,1)$ & $06 / 28(24,4)$ \\
Classificação & AIG & $09 / 29(31,1)$ & $04 / 31(12,9)$ & $13 / 60(21,7)$ \\
& PIG & $02 / 07(28,5)$ & $02 / 05(40,0)$ & $04 / 12(33,3)$ \\
& $1-3$ & $05 / 17(29,4)$ & $02 / 15(13,3)$ & $07 / 32(21,8)$ \\
Tempo VM & $4-7$ & $05 / 12(41,6)$ & $02 / 10(20,0)$ & $07 / 22(31,8)$ \\
(dias) & $>7$ & $01 / 07(14,2)$ & $02 / 11(18,1)$ & $03 / 18(16,6)$ \\
\hline
\end{tabular}

f/t: númerode falha / total de participantes no grupo

PN: peso de nascimento; IG: idade gestacional; VM: ventilação mecânica

Tabela 5 - Análise univariada, bruta e ajustada para peso de nascimento, idade gestacional, classificação e tempo de ventilação mecânica, do efeito da modalidade nIPPV sobre nCPAP na falha da extubação

\begin{tabular}{llll}
\hline & RR & IC 95\% & P \\
\hline Bruto & 0,55 & $0,23-1,32$ & 0,27 \\
Ajustado para PN & 0,47 & $0,18-1,23$ & 0,19 \\
Ajustado para IG & 0,49 & $0,20-1,24$ & 0,21 \\
Ajustado para Classificação & 0,57 & $0,24-1,34$ & 0,29 \\
Ajustado para tempo de VM & 0,56 & $0,23-1,40$ & 0,32 \\
\hline
\end{tabular}

RR: risco relativo; IC 95\%: intervalo de confiança de $95 \%$

PN: peso de nascimento; IG: idade gestacional;VM: ventilação mecânica. 
Tabela 6 - Análise multivariada, bruta e com ajuste de possíveis confundidores, do efeito das modalidades de ventilação não invasiva sobre a falha de extubação

\begin{tabular}{lllll}
\hline Análise & Variável & RC & IC 95\% & p Wald \\
\hline Bruta & nIPPV & 1.00 & & \\
& nCPAP & 2,2 & $0,71-6,79$ & 0,17 \\
Ajustada* & nIPPV & 1.00 & & \\
& nCPAP & 2,80 & $0,83-9,43$ & 0,097 \\
$\begin{array}{l}\text { Ajustada* e restrita a } \\
\text { RN } \geq \mathbf{1 0 0 0 g}(\mathbf{n}=\mathbf{6 0})\end{array}$ & nIPPV & 1.00 & & \\
\hline
\end{tabular}

RC: razão de chance; IC 95\%: intervalo de confiança de $95 \%$

*retirando a interferência do peso de nascimento, idade gestacional, classificação e tempo de ventilação mecânica

Gráfico 3 - Principais causas de falha da extubação nos 17 RNPT

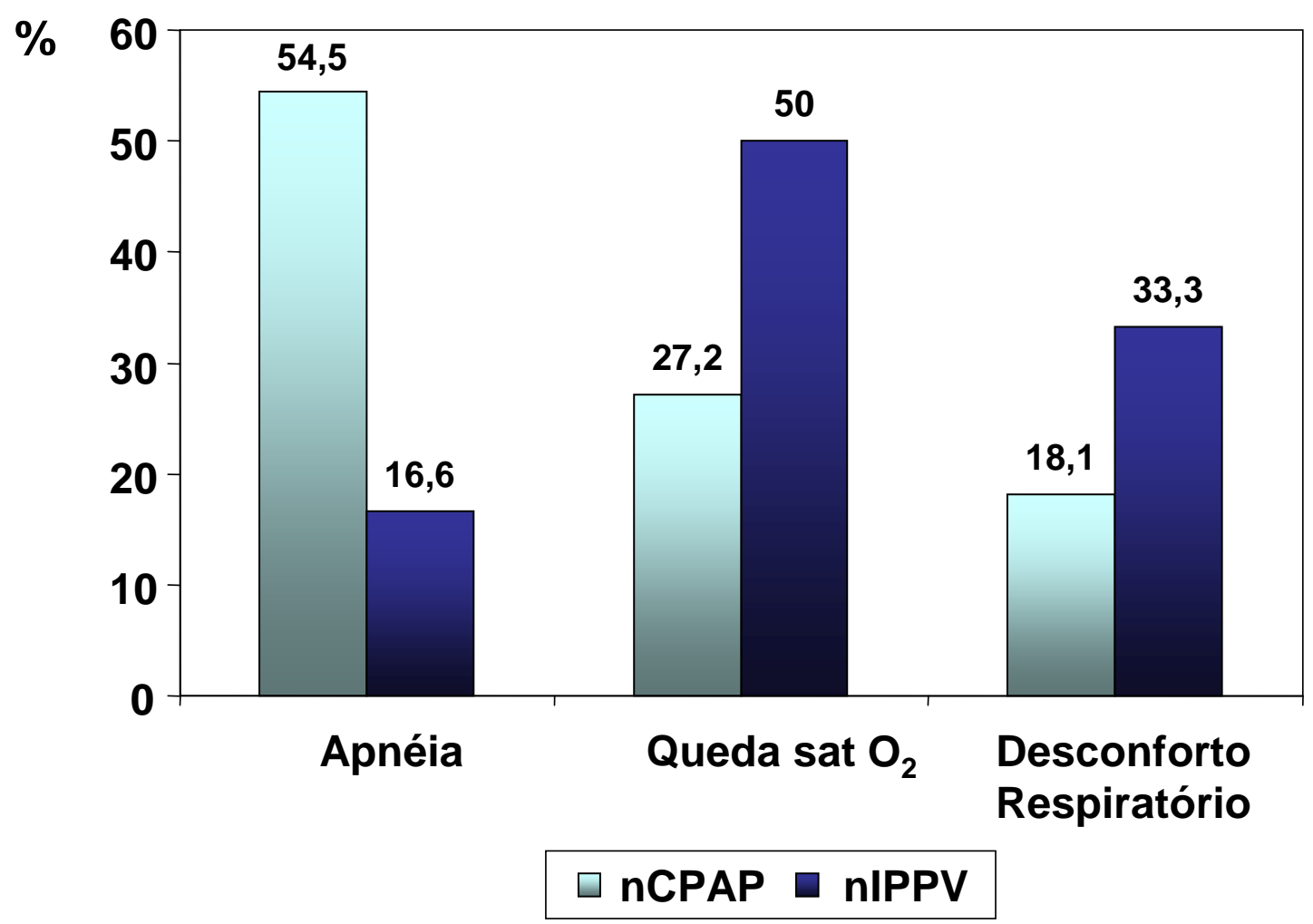


Tabela 7 - Total de casos e incidência de falha de extubação de acordo com o Hospital de origem

\begin{tabular}{llccc}
\hline & & HESA & ICr & P \\
\hline Ventilação por nCPAP, n (\%) & & $17(47,2)$ & $19(52,8)$ & 0,08 \\
Ventilação por nIPPV, n (\%) & & $09(25,0)$ & $27(75,0)$ & \\
\cline { 1 - 2 } Incidência de falhas, n/t (\%) & nCPAP & $6 / 17(35,2)$ & $5 / 19(26,3)$ & \multirow{2}{*}{0,45} \\
& nIPPV & $3 / 9(33,3)$ & $3 / 27(11,1)$ & \\
\hline
\end{tabular}

ICr: Instituto da Criança HESA: Hospital Estadual de Santo André

RR ajustado para tipo de hospital: 0,64 (IC95\%: 0,28 - 1,48) P=0,45

Tabela 8 - Resultados da Ultrassonografia de Crânio e de Abdome após a extubação de 72 RN submetidos à ventilação por nCPAP ou nIPPV

\begin{tabular}{cccccc}
\hline & \multicolumn{2}{c}{ nCPAP } & \multicolumn{4}{c}{ nIPPV } \\
\cline { 2 - 6 } & Normal & Alterado & Normal & Alterado & "P" \\
\hline $\begin{array}{c}\text { USG Crânio } \\
\mathbf{n ( \% )}\end{array}$ & $32(91,4)$ & $3(8,6)$ & $28(84,8)$ & $5(13,8)$ & 1,00 \\
$\begin{array}{c}\text { USG Abdome } \\
\mathbf{n ( \% )}\end{array}$ & $25(100)$ & - & $17(100)$ & - & 1,00 \\
\hline
\end{tabular}


5 - DISCUSSÃO 
Nas últimas décadas o número de $\mathrm{RN}$ de $\mathrm{MBP}$ que requerem ventilação mecânica prolongada tem aumentado e atualmente os desafios do neonatologista, não são mais os recém-nascidos de baixo peso, e sim, aqueles de extremo baixo peso (PN < 1000 gramas) (Darlow et al., 2003). Esforços para limitar a duração da VM têm sido direcionados para o desmame precoce do suporte ventilatório com a finalidade de diminuir não somente a incidência da mortalidade mas também da morbidade que é elevada nesta população de RN. Entretanto a extubação precoce desses RN tem sido acompanhada por várias dificuldades devido particularmente a instabilidade da caixa torácica, presença de atelectasias alveolares e dano pulmonar residual (Davis e Henderson-Smart, 2001).

Neste estudo comparamos duas técnicas de suporte ventilatório utilizadas após a extubação: a nCPAP, que é uma modalidade demonstrada previamente ser efetiva sendo frequentemente utilizada, e a nIPPV, que é uma abordagem relativamente recente no período neonatal e que pode contribuir na redução das complicações iatrogênicas da VM (Barrington et al., 2001).

Salientamos que a modalidade nIPPV foi utilizada no modo não sincronizado, de acordo com o tipo de aparelho de ventilação mecânica disponível nas Unidades Neonatais envolvidas no estudo. Na literatura há poucos estudos comparando a efetividade da nIPPV sincronizada sobre a não sincronizada na ventilação não invasiva do RNPT (Kugelman et al., 2007). 
Conforme descrito na casuística, entre os 72 RNPT estudados, 36 foram randomizados para receber nIPPV, e 36 para receber nCPAP. A média de peso ao nascimento foi de 1271 e 1425 gramas $(P=0,10)$, respectivamente; e a IG foi de 30,2 e 31,3 semanas $(P=0,043)$, respectivamente. No grupo randomizado para receber nCPAP a média de nota de Apgar no quinto minuto de vida foi de 7,7 e no grupo nIPPV foi de $8,0(P=0,36)$. Conforme pode ser visto na tabela 1 a distribuição das variáveis demográficas e de nascimento foram semelhantes, com exceção da idade gestacional que mostrou diferença significativa entre os dois grupos de estudo $(P=0,04)$. Os RN do grupo nIPPV apresentaram média de idade gestacional um pouco menor que os RN do grupo nCPAP.

Para evitar a interferência de potenciais confundidores comparamos as taxas de complicações comuns da prematuridade, tais como sepse neonatal, displasia broncopulmonar, persistência do canal arterial, enterocolite necrosante e hemorragia intracraniana anteriores à extubação. A incidência de PCA no grupo nIPPV foi percentualmente maior que no grupo nCPAP $(19,4$ vs 5,5 \%), não atingindo diferença estatística $(P=0,15)$. Do mesmo modo, os dois grupos (nCPAP e nIPPV) foram semelhantes também em relação a incidência de sepse, DBP e HIC, não apresentando diferença estatisticamente significante, conforme pode ser visto no gráfico 1. Ressaltamos que entre os dois grupos de estudo nenhum RN teve diagnóstico de enterocolite necrosante.

As evoluções clínica e ventilatória previamente à extubação foram semelhantes em ambos os grupos (tabela 2). A aminofilina foi utilizada em 
$91,6 \%$ das crianças que foram extubadas e randomizadas para a modalidade nCPAP, e em $86,1 \%$ daquelas que foram randomizadas para o grupo nIPPV, não mostrando diferença estatística entre os dois grupos. A grande maioria dos $\mathrm{RN}$ recebeu pelo menos uma dose de surfactante exógeno ( $80,5 \%$ no grupo nIPPV e $83,3 \%$ no grupo nCPAP, $P=1,00)$. $O$ tempo médio de permanência em ventilação mecânica (6,2 e 7,3 dias respectivamente, $\mathrm{P}=0,59)$, e a média da $\mathrm{FiO}_{2}$ pré-extubação $(29,9$ e 28,8 respectivamente, $P=0,71$ ) foram semelhantes entre os dois grupos.

Com relação ao tempo de uso das modalidades ventilatórias do estudo, a média foi de dois dias para o grupo nCPAP e de um dia e meio para o grupo nIPPV $(P=0,28)$. Igualmente ao tempo de uso das modalidades da assistência respiratória, o tempo de suporte de oxigênio inalatório após a suspensão das mesmas também foi semelhante, ou seja, 3,7 dias para o grupo nCPAP e 3 dias para o grupo nIPPV $(P=0,33)$. Em relação a análise dos gases sanguíneos, o valor do $\mathrm{pH}$ do grupo nIPPV foi inferior ao do grupo nCPAP $(7,34$ vs 7,38$)$. Embora esta diferença tenha sido estatísticamente significativa $(P=0,05)$, não apresenta significância clínica. Os valores da $\mathrm{PaCO}_{2}$ no primeiro dia pós-extubação também foram semelhantes em ambos os grupos, conforme pode ser visto na tabela 3 . Estudos prévios demonstraram valores similares nos níveis da $\mathrm{PaCO}_{2}$ entre as duas modalidades de estudo. Neste aspecto, Ryan et al. (1989) em seu estudo avaliaram 20 RNPT com IG inferior a 32 semanas que foram randomizados para receberem as modalidades nCPAP e nIPPV por um período de 6 horas após a extubação, e não encontrando diferença 
significante entre os valores da $\mathrm{PaCO}_{2}$ nos grupos de estudo. Da mesma forma, Lin et al. (1998) estudaram 34 RNPT com média de peso de nascimento de 1020 gramas e idade gestacional de 27,6 semanas, dos quais 18 foram randomizados para receberem nIPPV e 16 para nCPAP. Durante o período de estudo, de apenas 4 horas após a extubação, os autores também não encontraram diferença significativa nos valores da $\mathrm{PaCO}_{2}$ entre os grupos de estudo. Diferente do nosso estudo, Moretti et al. (1999) ao avaliarem 11 RN com média de peso de nascimento de 1141 gramas e de idade gestacional 28,1 semanas constataram que a $\mathrm{PaCO}_{2}$ foi mais baixa durante o uso da nIPPV (modo sincronizado) quando comparado ao uso da nCPAP, sendo esta diferença estatisticamente significativa. Devemos ressaltar que nesta pesquisa os $\mathrm{RN}$ foram estudados por um período apenas de 60 minutos após a extubação, tendo cada criança recebido as duas modalidades de estudo.

É consenso na literatura que a modalidade nIPPV pode causar complicações gastrointestinais devido a possibilidade da ocorrência de distensão gástrica levando a suspensão da alimentação, maior frequência de resíduos gástricos e até mesmo perfuração gástrica. Garland et al. (1985) avaliaram retrospectivamente 20 RNPT que apresentaram perfuração gástrica não associada a enterocolite necrosante ou obstrução intestinal, com a finalidade de determinar se o tipo de suporte ventilatório utilizado (ventilação através de tubo endotraqueal, de "prong" nasal ou de máscara facial) estava associado ou não a ocorrência de perfuração gástrica. Os autores constataram que a ventilação através do uso de máscara facial ou 
"prong" nasal favorecia um aumento no risco de perfuração gástrica de cerca de $30 \%$ quando comparada ao uso do tubo endotraqueal. No entanto, os próprios autores chamam atenção para algumas falhas possíveis do estudo, como seja um subdiagnóstico de enterocolite necrosante ou outra patologia do trato gastrointestinal as quais poderiam ter ocorrido. Chamaram a atenção ainda que a maioria dos RN participantes do estudo recebeu os dois tipos de ventilação (através de "prong" nasal e de tubo endotraqueal), e portanto a perfuração gástrica poderia ter ocorrido durante o período de ventilação com o uso de tubo traqueal e a sintomatologia ter se manifestado durante o uso do "prong" nasal ou da máscara facial. Relataram que cerca de $73,3 \%$ dos RN com perfuração gástrica tiveram asfixia moderada a grave (de acordo com a nota de Apgar no quinto minuto de vida), e portanto, poderiam ter sofrido isquemia do trato gastrointestinal facilitando a ocorrência de perfuração gástrica.

Em nosso estudo a ocorrência de resíduo gástrico ou de distensão abdominal que impedisse a alimentação foi semelhante entre os dois grupos de estudo, e nenhum dos pacientes participantes da nossa pesquisa apresentou perfuração gástrica. Kugelman et al. (2003) também não observaram a ocorrência de complicações gastrointestinais em seu estudo. Fatos semelhantes foram descritos por outros autores (De Paoli et al., Davis et al., Friedlich et al.,1999). Barrington et al., (2001) não demonstraram aumento da incidência de distensão abdominal ou intolerância alimentar nos 54 RN submetidos às duas modalidades de ventilação não invasiva, e também não constataram nenhum caso de perfuração gástrica. Fato 
semelhante foi descrito por Kiciman et al. (1998), dentre os 14 RNPT estudados.

Independente da modalidade de ventilação não invasiva utilizada em nosso a taxa de falência da extubação foi de $23,6 \%$, sendo este resultado semelhante àqueles encontrados na literatura. Barrington et al. (2001) em seu estudo controlado e randomizado, compararam a taxa de falência da extubação em 54 RN com peso de nascimento inferior a $1250 \mathrm{~g}$ e idade gestacional de 26,1 semanas por um período de 72 horas após a extubação utilizando as modalidades nCPAP e nIPPV, observando uma taxa de falência de 29,6\%. Entre os 64 RN estudados por Khalaf et al. (2001), cerca de $21,8 \%$ apresentaram falência da extubação durante o uso da ventilação não invasiva. Stefanescu et al. (2003), ao avaliarem a extubação em RNPT com peso de nascimento inferior a 1000 gramas constataram falência em $38,2 \%$ de seus pacientes.

Em nossa pesquisa a avaliação da falha de extubação entre os RN que foram randomizados para o grupo nIPPV e para o grupo nCPAP não mostrou diferença estatisticamente significativa. Conforme pode ser visto no gráfico 2, entre os $36 \mathrm{RN}$ randomizados para receberem a modalidade nIPPV, $6(16,6 \%)$ apresentaram falha de extubação em comparação a 11 $(30,5 \%)$ dos $36 \mathrm{RN}$ randomizados para nCPAP. Apesar de percentualmente a falha de extubação tenha sido quase o dobro nos RN em nCPAP que naqueles em nIPPV, e ter havido uma redução relativa no risco $(R R)$ de falência da extubação da ordem de 45 (IC95\%: 0,23 a 1,32), não foi possível 
demonstrar diferença estatisticamente significativa entre estas duas modalidades de ventilação não invasiva.

Verificamos em alguns estudos (Stefanescu et al., 2003; Barrington et al., 2001; Lesiuk et al., 2002) taxas variáveis em relação a falência da extubação, sendo por vezes associadas ao peso de nascimento e idade gestacional mais baixos (Dimitrou et al., 2002).

Deste modo procuramos verificar se havia relação entre a falência da extubação nas duas modalidades e o peso de nascimento, a idade gestacional, a classificação do RN e o tempo de ventilação mecânica.

Tendo em vista que os RNPT com peso de nascimento abaixo de 1250 gramas demonstram maior necessidade de suporte respiratório, bem como taxas significantes de morbidade e mortalidade (Davis et al., 2001), classificamos os RN em duas categorias de acordo com o peso de nascimento: $\mathrm{RN}<1250$ gramas e $\mathrm{RN} \geq 1250$ gramas para fins de análise estatística.

Constatamos que as taxas percentuais de falha da extubação nos RN com $\mathrm{PN}<1250$ gramas foram semelhantes entre as duas modalidades de ventilação não invasiva, e naqueles com $P N \geq 1250$ gramas a falha de extubação foi cerca de quatro vezes maior no grupo nCPAP quando comparado com o grupo nIPPV. No entanto a análise estatística dos dados não demonstrou diferença significativa. Fato similar foi também descrito por outros autores (Khalaf et al., 2001; Barrington et al., 2001). 
Em relação a falha de extubação de acordo com a idade gestacional, nos $R N$ com IG $<32$ semanas a taxa foi de $25 \%$, enquanto que no grupo com IG $\geq 32$ semanas foi de $21,4 \%$, para ambas as modalidades em geral.

Quando avaliados separadamente por grupo de estudo, esta característica se repetiu, ou seja, tanto no grupo nIPPV quanto no grupo nCPAP houve maior chance de falha entre os $\mathrm{RN}$ com IG $<32$ semanas (tabela 4). De acordo com Dimitrou et al. (2002) a falha de extubação pode estar associada a baixa força do músculo respiratório, que está relacionada com a maturidade ao nascimento. O uso da ventilação mecânica por período prolongado também interfere na força do músculo respiratório, semelhante a uma atrofia por desuso.

Ao se avaliar a falha da extubação em relação a classificação do RN observamos que no grupo nCPAP a maior taxa de falha ocorreu no grupo AIG $(31,1 \%)$ que no PIG $(28,5 \%)$, enquanto que no grupo nIPPV esta falha foi percentualmente maior no grupo PIG (40\%) que no grupo AIG (12,9\%). Salientamos que houve grande diferença entre o número de casos em cada grupo (60 RNPT AIG vs 12 RNPT PIG), não mostrando no entanto diferença significativa. Na literatura não encontramos nenhum estudo que tenha avaliado a falência da extubação em relação a classificação do RN.

Fato semelhante constatamos em relação ao tempo de VM prévio a extubação. O grupo nIPPV permaneceu em VM por um maior número de dias que o grupo nCPAP (7,3 vs 6,2$)$, não havendo diferença estatisticamente significativa $(P=0,59)$. Observamos ainda que o grupo que 
permaneceu em VM por um período de 4 a 7 dias apresentou maior taxa de falha da extubação.

Com relação ao tempo de uso das modalidades, este foi semelhante em ambos os grupos em relação às falhas, ou seja, 1,09 dias nCPAP e 1,16 dias em nIPPV.

Conforme descrito acima não houve diferença estatisticamente significativa entre a associação do peso de nascimento, a idade gestacional, a classificação, o tempo de ventilação mecânica e as modalidades de estudo, sendo que todas as estimativas ajustadas de Risco Relativo (nIPPV versus nCPAP) não se distanciaram muito do valor bruto de 0,55 (tabela 5).

Verificamos em nosso estudo que ao realizar uma regressão logística ajustada para o peso de nascimento, idade gestacional, classificação e tempo de ventilação mecânica a chance de falha na modalidade nCPAP aumenta de 2,2 para 2,8 vezes em relação a nIPPV. A probabilidade desse aumento ser atribuído ao acaso diminui, mas permanece na faixa de "tendência a significância estatística" $(P=0,097)$. Ao se restringir a análise, excluindo-se os RN de extremo baixo peso, ou seja peso de nascimento inferior a 1000 gramas, pudemos constatar um aumento para 4,38 vezes (IC $95 \% 1,03$ - 18,65) a chance de falha de extubação no grupo nCPAP quando comparado ao grupo nIPPV.

Khalaf et al. (2001) ao estudar RN com média de peso de nascimento em torno de 1000 gramas demonstraram uma superioridade da nIPPV (94\% sucesso) em relação a nCPAP (60\%), diferença esta que foi estatisticamente significante. Ao estudar RN com peso de nascimento abaixo de 1250 
gramas, Barrington et al. (2001) também constataram maior efetividade da modalidade nIPPV em relação a nCPAP, com taxa de falha da nIPPV de 14,8\% e da nCPAP de 44,4\%, diferença que também alcançou significância estatística.

O mecanismo que explica o fato da nIPPV ser mais benéfica ou efetiva que a nCPAP ainda não foi elucidado completamente. Moretti et al. (1999) consideram que a nIPPV pode aumentar a patência das vias aéreas superiores por criar uma pressão elevada na faringe e por promover inflação intermitente da faringe, ativando deste modo o ritmo respiratório. Em seu estudo os autores realizaram provas de função pulmonar e constataram que durante a nIPPV os volumes pulmonares (volume tidal e volume minuto) foram significativamente maiores em comparação com a nCPAP, e os valores da $\mathrm{PaCO}_{2}$ transcutânea também foram significativamente mais baixos em relação ao grupo nCPAP. Lin et al. (1998) também acreditam que a pressão intermitente criada na faringe é mais alta quando se utiliza a modalidade nIPPV, e que o fluxo de ar ao passar pela faringe pode ativar o músculo dilatador da faringe, estimulando a respiração e diminuindo os episódios de apnéia. De acordo com os autores, aumentos nas incursões da parede torácica e impulsos respiratórios foram observados quando os RN recebiam a modalidade nIPPV. Tais impulsos não foram notados quando os RN recebiam a modalidade nCPAP. Há evidências que estes impulsos tenham sido induzidos pela nIPPV, e seriam responsáveis em recrutar mais alvéolos desfazendo áreas de microatelectasias, o que justifica o seu sucesso (Kugelman et al., 2007). 
Em nosso estudo, não encontramos diferença significante entre o grupo nCPAP e o grupo nIPPV em relação aos fatores determinantes das falhas, ou seja: episódios recorrentes de apnéia (54,5\% vs 16,6\%), diminuições freqüentes da saturação de oxigênio $(27,2 \%$ vs $50 \%)$ e sinais clínicos de desconforto respiratório (18,1\% vs 33,3\%). Embora a ocorrência de apnéia no grupo nCPAP tenha sido numericamente maior em relação ao grupo nIPPV (6 vs 1), esta diferença não se mostrou significativa $(P=0,30)$.

A apnéia da prematuridade é definida como uma pausa respiratória com duração superior a 20 segundos ou de menor duração, se acompanhada de bradicardia e/ou cianose, e sua incidência é inversamente proporcional ao peso de nascimento e a idade gestacional (Barrington et al., 2001). Embora a sua etiologia não tenha sido ainda completamente elucidada, parece haver influência da imaturidade dos sistemas neurológico e respiratório, da resposta dos quimiorreceptores periféricos e centrais a hipóxia e hipercapnia, bem como a possibilidade de obstrução das vias aéreas, inadequada função dos músculos respiratórios e a participação de eventos ambientais e metabólicos (Barrington et al.,2001).

Em nossa pesquisa o principal fator relacionado a falha da extubação, independente do grupo de estudo, foi a apnéia, que esteve presente em $41,2 \%$ dos RN. Nos estudos de Barrington et al. (2001) a ocorrência de apnéia constituiu uma das causas principais de falência da extubação. $\mathrm{Na}$ pesquisa de Stefanescu et al. (2003) a ocorrência de apnéia e bradicardia foi responsável pela falha em $58 \%$ dos RN participantes do estudo. Do mesmo modo, Khalaf et al. (2001) relataram episódios de apnéia como responsáveis 
por $41 \%$ das falhas. Ao se avaliar a incidência de apnéia em relação ao grupo de estudo, os autores observaram que no grupo nCPAP a ocorrência de apnéia foi responsável por $46,7 \%$ das falhas enquanto no grupo nIPPV, $32,3 \%$, não mostrando diferença significante, semelhante aos nossos resultados. Com relação ao número de episódios de apnéia, os RN submetidos a nIPPV apresentaram menor número em relação ao grupo submetido a nCPAP, porém esta diferença também não foi significativa. Entre os três estudos avaliados por Davis et al. (2001) em sua metanálise, apenas um investigou a ocorrência de apnéia, cujos resultados mostraram uma tendência em reduzir os episódios de apnéia nos $\mathrm{RN}$ randomizados para a modalidade nIPPV, não sendo estatisticamente significante.

Quando realizamos a análise das proporções de modos de ventilação não invasiva e de falhas da extubação por tipo de Instituição participante no estudo verificamos efeito semelhante, ou seja, superioridade da modalidade nIPPV. Embora tenha havido maior taxa de falha na modalidade nCPAP no $\operatorname{ICr}(65,4 \%)$, o ajuste do risco relativo de falhas mostrou mudança pequena no $\operatorname{RR}$ (de 0,55 para 0,64), diferença esta que não alcançou significância estatística $(P=0,45)$.

A maioria dos RN participantes do estudo realizou USG de crânio após a extubação. Entre os 33 pacientes do grupo nIPPV, 28 (84,8\%) apresentaram resultados normais, e $5(15,2 \%)$ resultado alterado, mas esta diferença não foi estatisticamente significante. As alterações encontradas foram: aumento difuso e simétrico do parênquima encefálico (atribuído a prematuridade), hemorragia intracraniana grau 1, hemorragia retroventricular 
e da região subpendimária esquerda, área isquêmica com porencefalia na região frontal esquerda e hiperecogenicidade da coroa radiata, e leve dilatação dos ventrículos laterais. Com relação ao grupo nCPAP, 35 RN realizaram USG de crânio, sendo que em 32 (91,4\%) os resultados foram normais e $3(8,6 \%)$ apresentaram as seguintes alterações: leve dilatação do terceiro ventrículo, sinais de hemorragia intracraniana grau 1 com discreta dilatação ventricular, e presença de leucomalácia nas regiões periventriculares bilateral (alteração que já existia previamente a extubação).

Como pode visto na tabela 8 , os resultados da Ultrassonografia de Crânio após a extubação não mostraram diferença estatisticamente significante entre os dois grupos de estudo.

A maioria dos $\mathrm{RN}$ foi avaliada através da realização de ultrassonografia de abdome, e não foram encontradas quaisquer alterações (tabela 8).

Durante o período de 72 horas do nosso estudo, quer na modalidade nIPPV ou na modalidade nCPAP, não foram observadas a ocorrência de efeitos colaterais como necrose de septo nasal, obstrução do "prong" nasal entre outras. Fato semelhante também foi observado por outros autores (Lin et al., 1998; Khalaf et al., 2001; Barrington et al., 2001; Stefanescu et al., 2003). A tração exercida pelo peso do "prong" acoplado a narina do RN é um fator de risco para a ocorrência de lesão nasal.

Durante a internação alguns dos RN que apresentaram falha da extubação quando em uso da modalidade nCPAP, foram colocados na modalidade nIPPV na tentativa de se evitar a re-intubação orotraqueal, e 
observamos que estes RN tiveram boa resposta em uso desta modalidade, evitando-se a re-intubação orotraqueal. Na literatura encontramos relato de apenas um estudo que procedeu da mesma forma, e os autores também constataram que muitos pacientes que apresentaram falência da extubação em uso da modalidade nCPAP puderam ser resgatados com sucesso com o uso da modalidade nIPPV (De Paoli et al., 2003).

De forma semelhante a outros estudos (Lin et al., 1998; Kiciman et al., 1998; Moretti et al., 1999; Barrington et al., 2001; Khalaf et al., 2001) nenhuma criança de nossa pesquisa foi a óbito durante o uso das modalidades de ventilação não invasiva.

Barrington et al. (2001) e Davis et al. (2001) foram unânimes ao afirmar que a nIPPV é um método útil e eficaz em aumentar os benefícios da nCPAP na prevenção da falência da extubação dos RNPT nas primeiras horas após a extubação.

Do mesmo modo, Khalaf et al. (2001) consideram a nIPPV como uma modalidade mais efetiva que a nCPAP no desmame do RN com SDR em uso de ventilação mecânica, e recomendam que a nIPPV deva ser utilizada como modo primário de extubação mesmo naqueles $\mathrm{RN}$ com função pulmonar prejudicada.

Na medida em que a ventilação mecânica possa ser evitada, através da utilização da ventilação não invasiva, particularmente a nIPPV, haverá uma diminuição na incidência de seqüelas incapacitantes como a displasia broncopulmonar e a hemorragia cerebral, melhorando não apenas a sobrevida, mas a qualidade de vida destes pacientes. 
6 - CONCLUSÃO 
Tendo em vista os nossos resultados concluímos que:

1. Embora a distribuição de freqüências de falha tenha mostrado numericamente um menor índice de falha nos RNPT submetidos à nIPPV em relação àqueles submetidos à nCPAP, não houve diferença estatísticamente significante entre os dois modos de suporte ventilatório após a extubação.

2. A associação entre o peso de nascimento, a idade gestacional, a classificação do RN e o tempo de ventilação mecânica e a freqüência de falha na extubação não se mostrou estatisticamente significante.

3. Constatamos uma chance de falha de extubação de 4,38 vezes maior no grupo nCPAP quando comparado ao grupo nIPPV após excluírmos os RN com peso de nascimento inferior a 1000 gramas, sendo esta diferença estatisticamente significativa $(P=0,045)$.

4. Complicações gastrointestinais e neurológicas, além de outras complicações, não ocorreram nos recém nascidos pré-termo submetidos à nIPPV ou nCPAP após a extubação. 
7 - ANEXOS 
ANEXO A

TERMO DE CONSENTIMENTO LIVRE E ESCLARECIDO 
HOSPITAL DAS CLÍNICAS DA FACULDADE DE MEDICINA DA USP HOSPITAL ESTADUAL MÁRIO COVAS - SA / FACULDADE DE MEDICINA DO ABC

TERMO DE CONSENTIMENTO LIVRE E ESCLARECIDO

\section{I - DADOS DE IDENTIFICAÇÃO DO SUJEITO DA PESQUISA OU RESPONSÁVEL LEGAL}

1. NOME DO PACIENTE

DOCUMENTO DE IDENTIDADE N':

DATA NASCIMENTO: .......................

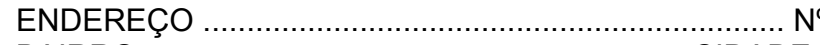

BAIRRO: CIDADE

CEP TELEFONE: DDD

$(\ldots \ldots \ldots .$.

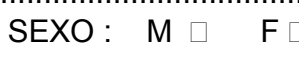

APTO:

\section{.}

2.RESPONSÁVEL LEGAL

NATUREZA (grau de parentesco, tutor, curador etc.) DOCUMENTO DE IDENTIDADE :......

SEXO: $M \quad \square \quad F$

DATA NASCIMENTO.: .....................

ENDEREÇO:

$\mathrm{N}^{\circ}$

CIDADE:

CEP:

TELEFONE: DDD (............)

\section{II - DADOS SOBRE A PESQUISA CIENTÍFICA}

1. TÍTULO DO PROTOCOLO DE PESQUISA: ANÁLISE COMPARATIVA ENTRE O USO DE nIPPV (NASAL INTERMITTENT POSITIVE PRESSURE VENTILATION) E ICPAP (NASAL CONTINUOUS POSITIVE AIRWAY PRESSURE) EM RECÉM-NASCIDOS PRÉ-TERMO APÓS EXTUBAÇÃO TRAQUEAL.

2. PESQUISADOR: Edna Maria de Albuquerque Diniz CARGO/FUNÇÃO: Professor Livre Docente em Neonatologia do Departamento de Pediatria da FMUSP INSCRIÇÃO CONSELHO REGIONAL NNo 19327

UNIDADE DO HCFMUSP:Unidade de Cuidados Intensivo Neonatal (UCINE) - ICr

3. AVALIAÇÃO DO RISCO DA PESQUISA:

SEM RISCO ( ) RISCO MÍNIMO (X)

RISCO BAIXO( ) RISCO MAIOR ( )

RISCO MÉDIO ( )

(probabilidade de que o indivíduo sofra algum dano como conseqüência imediata ou tardia do estudo)

4.DURAÇÃO DA PESQUISA : 1 ano 


\section{III - REGISTRO DAS EXPLICAÇÕES DO PESQUISADOR AO PACIENTE OU SEU REPRESENTANTE LEGAL SOBRE A PESQUISA, CONSIGNANDO:}

1- justificativa e os objetivos da pesquisa - Prezado(a) Senhor(a), como já foi the falado seu filho nasceu antes da data prevista e apresenta dificuldade para respirar sozinho, pois seu pulmão ainda não se desenvolveu completamente. No momento ele se encontra respirando com o auxílio de um aparelho e uma cânula (que entra pela boca e chega até quase o pulmão) para levar o oxigênio diretamente ao pulmão. Quando ele estiver melhor, nós vamos retirar a cânula da boca e ajudá-lo a respirar através de um tubinho que fica na ponta do nariz. Nós observaremos o seu filho durante três dias, neste período nós iremos colher exames de sangue, realizar radiografias de tórax e abdome e ultrassonografia de abdome para avaliar se está tudo bem com a criança. Esses exames são feitos em todas as crianças normalmente, mesmo quando não estejam participando de algum trabalho; são realizados aqui mesmo no berçário. O sangue é colhido com agulhas e seringas descartáveis (jogados fora após o uso). No local da picada da agulha pode se formar um pequeno hematoma (mancha roxa), mas que desaparece em poucos dias, e o seu filho poderá sentir um pouco de dor na hora da coleta. Nós queremos saber se esse tipo de auxílio na respiração (através dos tubos nariz) é melhor para as crianças no sentido de elas conseguirem respirar melhor e não terem que voltar a ter que usar a cânula que entra pela boca. Se o(a) senhor(a) não quiser que seu filho participe da pesquisa tem todo o direito de se recusar a ela, e seu filho receberá assim mesmo o tratamento mais adequado para a sua doença. Caso ele comece na pesquisa e depois o(a) senhor(a) deseje desistir, também está garantido o melhor tratamento disponível no hospital para ele.

2- procedimentos que serão utilizados e propósitos, incluindo a identificação dos procedimentos que são experimentais - avaliação clínica ( através de exame físico) e laboratorial ( através de exames de sangue, Raio $X$ e ultrassonografia) dos RN, acompanhamento clínico após a retirada da criança do aparelho que auxilia na respiração em determinada técnica a ser definida por sorteio e posterior acompanhamento no ambulatório do Instituto da Criança.

3- desconfortos e riscos esperados - Espera-se apenas o desconforto da coleta dos exames de sangue, que podem ser um pouco doloridos e talvez deixar um pequeno hematoma (mancha roxa) no local da coleta, que desaparece rápido. Lembrar que de todas as crianças que estão internadas são colhidos exames rotineiros para melhor acompanhamento.

4-- benefícios que poderão ser obtidos - estudar a melhor forma de se conduzir o RN após a retirada da criança do aparelho que auxilia na respiração, assim ele terá menos chance de piorar da parte respiratória e ter que voltar para o aparelho de respiração artificial. 
5- procedimentos alternativos que possam ser vantajosos para o indivíduo - comprovar se esse novo tipo de acompanhamento do RN é bom para as crianças evitando que ele tenha que voltar a usar a cânula pela boca.

\section{IV - ESCLARECIMENTOS DADOS PELO PESQUISADOR SOBRE GARANTIAS DO SUJEITO DA PESQUISA:}

1. acesso, a qualquer tempo, às informações sobre procedimentos, riscos e benefícios relacionados à pesquisa, inclusive para esclarecer eventuais dúvidas - sim

2. liberdade de retirar seu consentimento a qualquer momento e de deixar de participar do estudo, sem que isto traga prejuízo à continuidade da assistência - sim

3. salvaguarda da confidencialidade, sigilo e privacidade - sim

4. disponibilidade de assistência no HCFMUSP, por eventuais danos à saúde, decorrentes da pesquisa - sim

5. viabilidade de indenização por eventuais danos à saúde decorrentes da pesquisa.

\section{INFORMAÇÕES DE NOMES, ENDEREÇOS E TELEFONES DOS RESPONSÁVEIS PELO ACOMPANHAMENTO DA PESQUISA, PARA CONTATO EM CASO DE INTERCORRÊNCIAS CLÍNICAS E REAÇÕES ADVERSAS.}

Edna Maria de Albuquerque Diniz

Telefones: 288-1117 (residencial) / 9979-1711 (celular)

Daniela Franco Rizzo

Telefones: 6940-4243 (residencial) / 9770-7614 (celular)

UCINE : 3069-8590 / HESA :6829-5109

\section{OBSERVAÇÕES COMPLEMENTARES:}


VII - CONSENTIMENTO PÓS-ESCLARECIDO

Declaro que, após convenientemente esclarecido pelo pesquisador e ter entendido o que me foi explicado, consinto em participar do presente Protocolo de Pesquisa

São Paulo, de de 2 ..........

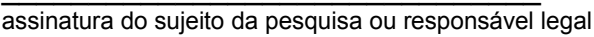

assinatura do pesquisador (carimbo ou nome Legível) 
ANEXO B

\section{PROTOCOLO DO ESTUDO}




\begin{tabular}{|c|c|c|c|c|}
\hline \multirow{2}{*}{\multicolumn{5}{|c|}{$\begin{array}{l}\text { HOSPITAL DAS CLÍNICAS DA FACULDADE DE MEDICINA DA USP } \\
\text { HOSPITAL ESTADUAL MÁRIO COVAS - SA I FACULDADE DE MEDICINA DO } \\
\text { ABC } \\
\text { PROTOCOLO: ANÁLISE COMPARATIVA ENTRE O USO DE IIPPV (NASAL-VENTILAÇÃO POR } \\
\text { PRESSÃO POSITIVA INTERMITENTE) E nCPAP (NASAL-PRESSÃO POSITIVA CONTÍNUA EM } \\
\text { VIAS AÉREAS) EM RECÉM-NASCIDOS PREE-TERMO APÓS EXTUBAÇÃO TRAQUEAL. }\end{array}$}} \\
\hline & & & & \\
\hline \multicolumn{5}{|l|}{ IDENTIFICAÇÃO } \\
\hline \multirow{2}{*}{$\begin{array}{l}\text { Nome: } \\
\text { Data de Nascimento: } \\
\text { Data de Admissão: / / }\end{array}$} & & \multicolumn{3}{|c|}{ Prontuário: } \\
\hline & I / Idade: & dias & $\begin{array}{l}\text { Sexo: } \quad M \quad \quad F \square \\
\text { Procedência: }\end{array}$ & Cor: \\
\hline
\end{tabular}

\section{ANTECEDENTES}

a) Materno (pessoal) e obstétricos:

\begin{tabular}{|c|c|c|c|c|c|}
\hline $\begin{array}{l}\text { Idade: } \\
\text { Pré-Natal. }\end{array}$ & Gesta: & $\begin{array}{l}\text { Para: } \\
\mathrm{N}^{\circ} \text { consultas: }\end{array}$ & & & Idade Gestacional: \\
\hline Intercorrências:S $\square \mathbf{N}$ & Tipo: & $\square$ febre $\quad \square$ corri & $\begin{array}{l}\square \text { exantema } \\
\text { ento vaginal }\end{array}$ & $\square$ DST & $\square$ disúria \\
\hline
\end{tabular}

Parto: Local:

Tipo:

Intercorrências:

$\mathrm{N} \square$
$\mathrm{N}$
$\square$

$\mathrm{C} \square \quad \mathrm{F} \square$

Ruptura Membrana: No ato

Precoce $\square \quad$ Tempo:

b) Recém-nascido:

Peso Nascimento:

Capurro/Dubowitz:

Comp.:

PC:

Classificação:

Apgar:

Diagnósticos:

IOT:

Parâmetros da VM: PIP:

Fluxo:

PEEP:

Ri/e:

FR:

MAP:

FiO2:

Tratamento realizado: Surfactante prévio

S

N

$\neg$ Profilático

Rescue 
EXAME FÍSICO À ADMISSÃO

\begin{tabular}{lllll}
\hline IC: & IG: & Peso: & PC:
\end{tabular}

Exame Físico Geral:

Cabeça e Pescoço:

Ap. Respiratório:

AP. Cardiovascular:

Abdome:

Genito-urinário:

Exame Neurológico:

Pele:

Outras anormalidades:

Parâmetros da VM: PIP
Fluxo:
PEEP:
$R$ i/e:
FR:
MAP:
FiO2:

\section{DIAGNÓSTICOS}

EXTUBAÇÃO

data: 1 / horário:

IC:

IG:

Aminofilina:

VM PIP: PEEP: FR: FiO2: Fluxo: Ti: Ri/e: MAP:

Modo de Ventilação:

nIPPV

nCPAP

Gasometria: $\mathrm{pH}: \quad \mathrm{pCO}_{2}$ :

$\mathrm{pO}_{2}$ :

$\mathrm{HCO}_{3}:$

BE: $\quad \mathrm{FiO} 2$ : 
EVOLUÇÃO CLÍNICA:

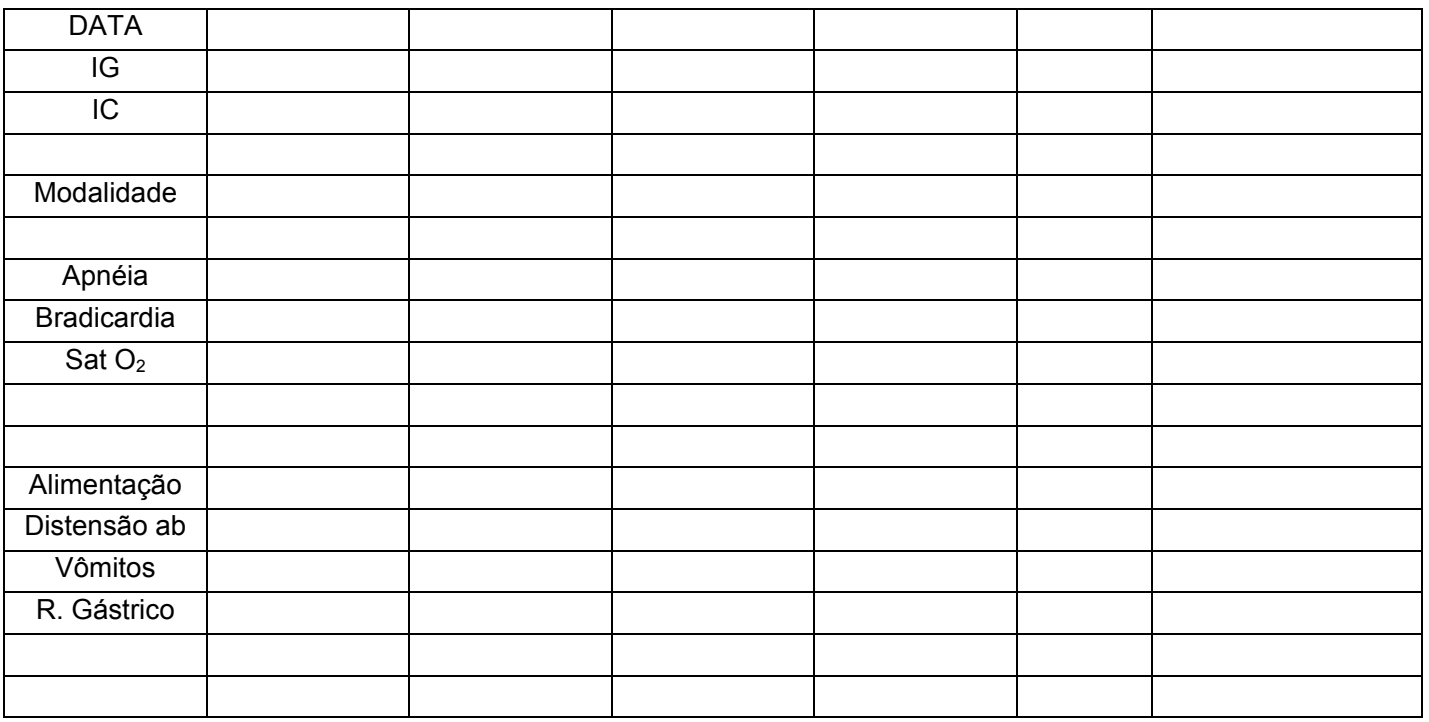

Suspensão Modalidade:

Suspensão $\mathrm{O}_{2}$

EXAMES COMPLEMENTARES

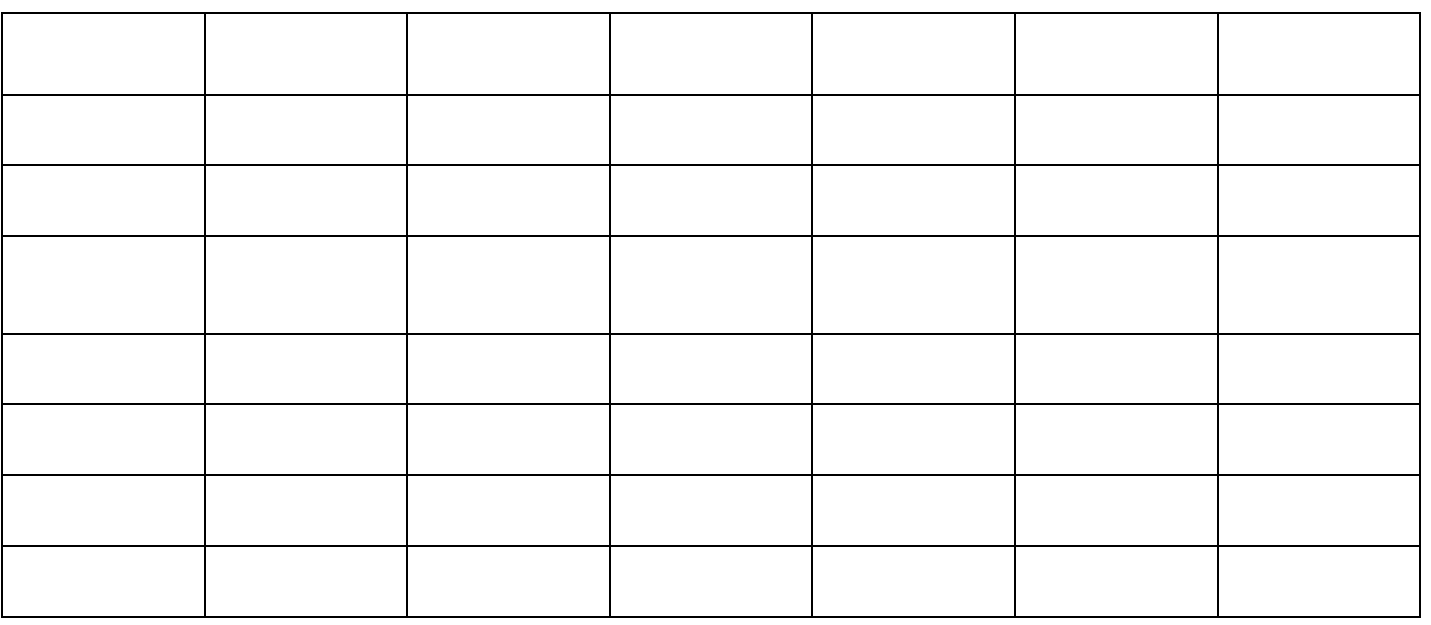


8 - REFERÊNCIAS BIBLIOGRÁFICAS 
Alexander GR, Himes JH, Kaufman RB, Mor J, Kogan M. A United States National reference for Fetal Growth. Obstetrics \& Gynecology. 1996; $87(2) ; 163-8$.

American Academy of Pediatrics, care of the neonate. In: Guidelines for perinatal care. $5^{\text {th }}$ ed. AAP; 2002;20;305-20.

Antunes LCO, Calixto CA, Oliveira CN, Saskia EPR, Rugolo LMSS. Failure to extubate in premature neonates of neonatal intensive care. Salusvita. 2003;22(3);395-404.

Ballard JL, Khoury JC, Wedig K, Wang L, Eilers-Walsman BL, Lipp R. New Ballard score, expanded to include extremely premature infants. Journal of Pediatrics. 1991;119:417-23.

Bancalari E, Del Moral T. Bronchopulmonary dysplasia and surfactant. Bio Neonate. 2001;80(1);7-13.

Barrington KJ, Bull D, Finer NN. Randomized trial of nasal synchronized intermittent mandatory ventilation compared with continuous positive airway pressure after extubation of very low birth weight infants. Pediatrics. 2001;107(4);638-41.

Barrington K, Finer N. The natural history of the appearance of apnea of prematurity. Pediatr Res. 1991;29;372-5.

Capurro H, Konichezky S, Fonseca D, Caldeuro-Barcia R. A simplified method for diagnosis of gestacional age in the newborn infant. J Pediatr. 1978;93;120-122. 
Darlow BA, Cust AE, Donoghue DA. Improved outcomes for very low birthweight infants: evidence from New Zealand national population based data. Arch. Dis. Child Fetal Neonatal. 2003;88;F23-F28.

Davis PG, Hendderson-Smart DJ. Post-extubation prophylactic nasal continuous positive airway pressure in preterm infants: Systematic review and meta-analysis. Pediatr Pulmonol. 1998;26(5);349-53.

Davis PG, Lemyre B, De Paoli AG. Nasal intermittent positive pressure ventilation (NIPPV) versus nasal continuous positive airway pressure (NCPAP) for preterm neonates after extubation (Cochrane Review). The Cochrane Library. 2001(3).

Davis PG, Hendderson-Smart DJ. Nasal continuous positive airways pressure immediately after extubation for preventing morbidity in preterm infants (Cochrane Review). The Cochrane Library. 2001(3).

De Klerk AM, De Klerk RK. Nasal continuous positive airway pressure and outcomes of preterm infants. J Paediat Child Healt. 2001;37;61-7.

De Paoli AG, Davis PG, Lemyre B. Nasal continuous pressure versus nasal inttermitent posiitve pressure ventilation for preterm neonates: a systematic review and meta-analysis. Acta paediatr. 2003;92;70-5.

Dimitrou G, Greenough A, Endo A, Cherian S, Rafferty GF. Prediction of extubation failure in preterm infants. Arch dis Child Fetal Neonatal. 2002; 82; F32-5.

Diniz EMA; Vaz FAC. Doença das Membranas Hialinas. Pediatria Moderna. 2000;36;5-11. 
Dries DJ. Weaning from mechanical ventilation. The Journal of Trauma: Injury, Infection, and Critical Care.1997;43(2);372-84.

Edward D, Dyer W, Northway W. Twelve years experience with bronchopulmonary dysplasia. Pediatrics. 1977;59;839-46.

Garland JS, Nelson DB, Rice T, Neu J. Increased risk of gastrointestinal perforation in neonates mechanically ventilated with either face mask or nasal prongs. Pediatrics. 1985;76;406-10.

Guinsburg R, Miyoshi M. Distúrbios respiratórios no período neonatal. São Paulo: Atheneu, 1998.

Halliday HL. Towards earlier neonatal extubation. The Lancet. 2000;355; 2091-2.

Henderson-Smart DJ, Davis PG. Prophylatic methylxantine for extubation in preterm infants (Cochrane Reviw). In: The Cochrane Library.1998; 4.

Horbar J, MCauliffe T, Adler S. Variability in 28-day outcomes for very low birth weight infants: an analysis of 11 neonatal intensive care units. Pediatrics.1988;82;554-9.

Jeena $\mathrm{P}$, Pillay $\mathrm{P}$, Adhikari $\mathrm{M}$. Nasal CPAP in newborns with acute respiratory failure. Annals of tropical Paediatrics. 2002;22;201-7.

Jobe AH, Bancalari E. Workshop Summary Bronchopulmonary Dysplasia. Am J Respir Crit Care Med. 2001;163;1723-9.

Kavvadia V, Greenough A, Dimitrou G. Prediction of extubation failure in preterm neonates. Eur J Pediatr. 2000;159;227-31. 
Khalaf MN, Brodsky N, Hurley J, Bhandari V. A prospective randomized, controlled trial comparing synchronized nasal intermittent positive pressure ventilation versus nasal continuous positive airway pressure as modes of extubation. Pediatrics. 2001;108(1);13-7.

Khan N, Brown A, Venkataraman ST. Predictors of extubation sucess and failure in mechanically ventilated infants and children. Pediatric Critical Care. 1996;24(8);1568-79.

Kiciman NM, Andreasson B, Bernstein G. Thoracoabdominal motion in newborns during ventilation delivered by endotracheal tube or nasal prongs. Pediatr Pulmonol. 1998;25;175-81.

Kugelman A, Feferkorn I, Riskin A, Chistyakov I, Kaufman B, Bader D. Nasal Intermittent Mandatory Ventilation Versus Nasal Continuous Positive Airway Pressure for Respiratory Distress Syndrome: A Randomized, Controlled, Prospective Study. The Journal of Pediatrics. 2007;521-7.

Kumar P, Kiran PS. Changing trends in the management of respiratory distress syndrome. The Indian Journal of Pediatrics. 2004;71(1);49-54.

Lemyre B, Davis PG, De paoli AG. Nasal intermittent positive pressure ventilation (NIPPV) versus nasal continuous positive airway pressure (NCPAP) for apnea of prematurity. (Cochrane Review). The Cochrane Library, 2000.

Lesiuk W, Lesiuk L, Maliczowska M, Puzniac G. Non invasive mandatory ventilation in extremely low birth weight and very low birth weight newborns with failed extubation. Przegl Lek. 2002;59(1);57-9. 
Lin $\mathrm{CH}$, Tsay WH, Lin YJ, Wang ST, Yeh TF. Efficacy of nasal intermittent positive pressure ventilation in treating apnea of prematurity. Pediatr Pulmonol. 1998;26;349-53.

Mador MJ, Mador M. Weaning from mechanical ventilation: What have we learned and what do we still need to know? Chest. 1998;114(3);672-4.

Malik RK, Gupta RK. A Two Year Experience in Continuous Positive Pressure Ventilation Using Nasal prongs and Pulse Oximetry. MJAFI. 2003;59;36-9.

Millar D, Kirpalani H. Benefits of Non Invasive Ventilation. Indian Pediatrics. 2004;41;1008-16.

Miller MJ, DiFiore JM, Strohl KP, Martin RJ. Effects of nasal CPAP on supraglottic and totalpulmonary resistance in preterm infants. $J$ Appl Physiol. 1990;168;141-6.

Moretti C, Gizzi C, Papoff P. Comparing the effects of nasal synchronized intermittent positive pressure ventilation (nSIPPV) and nasal continuous positive airway pressure (nCPAP) after extubation in very low birth weight infants. Early Hum Dev. 1999;56;167-77.

Morley C. Continuous distending pressure. Arch Dis Child. 1999;81;15267.

Muller M, Wagner CL, Annibale DJ, Hulsey TC, Knapp RG, Almeida JS. Web-based prediction of extubation outcome in premature infants on mechanical ventilation using an artificial neural network. 2003;945. 
Papile LA, Burstein R, Koffler $H$. Incidence and evolution of subependymal and intraventricular hemorrhage: a study of infants with weight less than 1500 grams. J Pediatr. 1978; 92; 529-34.

Parker MM. Predicting success of extubation in children. Crit. Care Med. 1996;104(4);1429-30.

Page NE, Giehl M, Luke S. Intubation complications in the critically ill child. American Association of Critical Care Nurses.1998;9(1);25-35.

Ramanathan R. Early surfactant therapy and noninvasive ventilation. Journal of Perinatology. 2007;27;S33-37.

Ryan CA, Finer NN, Peters KL. Nasal intermittent positive-pressure ventilation offers no advantages over nasal continuous positive airway pressure in apnea of prematurity. Am J Dis Child. 1989;143;1196-8.

Rugolo LMSS. Síndrome do desconforto respiratório. In: Departamento de Pediatria da Faculdade de Medicina da UNESP - Botucatu. Condutas em Pediatria. 2. ed. Rio de Janeiro:EPUB, 1999; 180-186.

Stefanescu BM, Murphy WP, Hansell BJ. A Randomised, Controlled trial comparing two different Continuous positive airway pressure systems for successful extubation of extremely low birth weight infants. Pediatrics. 2003;112;1031-8.

Subramaniam P, Hendderson-Smart DJ, Davis PG. Prophylactic nasal continuous positive airway pressure for preventing morbidity and mortality in very preterm infants. (Cochrane Review). The Cochrane Library, 3, 2001. 
Tomminska V, Heinonen K, Lehtonen L, Renlund M, Saarela T, Tamela O, Virtanen M, Fellman V. No Improvement in Outcome of Nationwide Extremely Low Birthweight Infant Populations Between 1996-1997 and 1999-2000. Pediatrics. 2007;119(1);29-36.

Tooley, WH. Epidemiology of bronchopulmonary dysplasia. J. Pediat. 1979;95;851-5.

Van Lierde S, Smith J, Devlieger H, Eggermont E. Outcome of respiratory distress syndrome at 28 days: a prospective longitudinal study. Eur Respir J. 1992;5;1243-8.

Venkataraman ST, Khan N, Brown A. Validation of predictors of extubation success and failure in mechanically ventilated infants and children. Critical Care Medical. 2000; 28; 2991-6.

Visveshwara N, Caliwag W, Peck M. Feasibilityof early extubation in RDS with application of nasal ventilation, as predicted by end-expiratory resistance. Pediatr Res. 1996;39;355 A. 
ANEXO C - Tabela comparativa entre os principais estudos encontrados na literatura

\begin{tabular}{|c|c|c|c|c|c|c|}
\hline AUTORES & CASUÍSTICA & MODALIDADE & FALÊNCIA & $\begin{array}{l}\text { PERÍODO DE } \\
\text { ESTUDO }\end{array}$ & $\begin{array}{l}\text { EFEITOS } \\
\text { COLATERAIS }\end{array}$ & CONCLUSÕES \\
\hline Ryan et al., 1989 & $\begin{array}{l}20 \mathrm{RN} \\
\text { IG } 26,2 \\
\text { PN } 1120\end{array}$ & $\begin{array}{l}\text { nCPAP } \\
\text { nIPPV }\end{array}$ & NA & 6 horas & Ausentes & 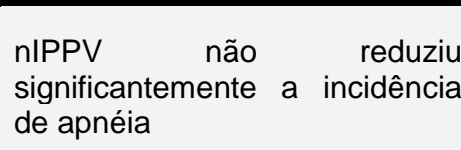 \\
\hline Lin et al., 1998 & $\begin{array}{l}34 \text { RN } \\
\text { IG } 27,6 \\
\text { PN } 1021\end{array}$ & $\begin{array}{l}\text { nCPAP } \\
\text { nIPPV }\end{array}$ & $6 \%$ & 4 horas & Ausentes & $\begin{array}{l}\text { nIPPV diminuiu a incidência de } \\
\text { apnéia e bradicardia de maneira } \\
\text { significativa. }\end{array}$ \\
\hline Kiciman et al., 1998 & $\begin{array}{l}14 \text { RN } \\
\text { IG } 30 \\
\text { PN } 1413\end{array}$ & $\begin{array}{l}\text { CPAP traqueal } \\
\text { nCPAP } \\
\text { nIPPV }\end{array}$ & NA & ND & Ausentes & $\begin{array}{l}\text { nIPPV pode ser uma alternativa } \\
\text { de tratamento para RNPT que } \\
\text { necessitam de VM }\end{array}$ \\
\hline Moretti et al., 1999 & $\begin{array}{l}11 \mathrm{RN} \\
\text { IG } 28,1 \\
\text { PN } 1141\end{array}$ & $\begin{array}{l}\text { nCPAP } \\
\text { nSIPPV }\end{array}$ & NA & 60 minutos & Ausentes & $\begin{array}{l}\text { nSIPPV mais eficaz em manter } \\
\text { a ventilação e reduzir os } \\
\text { esforços respiratórios. }\end{array}$ \\
\hline Khalaf et al., 2001 & $\begin{array}{l}64 \text { RN } \\
\text { IG } 28 \\
\text { PN } 1060\end{array}$ & $\begin{array}{l}\text { nCPAP } \\
\text { nSIPPV }\end{array}$ & $\begin{array}{l}40 \% \\
6 \%\end{array}$ & 72 horas & Ausentes & $\begin{array}{l}\text { nSIPPV mais efetiva no } \\
\text { desmame da VM }\end{array}$ \\
\hline Barrington et al.,2001 & $\begin{array}{l}54 \text { RN } \\
\text { IG 26,1 } \\
\text { PN } 840\end{array}$ & $\begin{array}{l}\text { nCPAP } \\
\text { nIPPV }\end{array}$ & $\begin{array}{l}44,4 \% \\
14,8 \%\end{array}$ & 72 horas & Ausentes & $\begin{array}{llll}\text { nIPPV mais eficaz } & \text { na } \\
\text { diminuição da incidência } & \text { de } \\
\text { apnéia, e mais efetiva } & \text { na } \\
\text { prevenção da falência da } & \text { da } \\
\text { extubação. } & \end{array}$ \\
\hline Komatsu, et al., 2007 & $\begin{array}{l}72 \text { RN } \\
\text { IG } 30,6 \\
\text { PN } 1348\end{array}$ & $\begin{array}{l}\text { nCPAP } \\
\text { nIPPV }\end{array}$ & $\begin{array}{l}30,5 \% \\
16,6 \%\end{array}$ & 72 horas & Ausentes & $\begin{array}{l}\text { chance de falha de extubação } \\
\text { estatisticamente significante } \\
\text { maior no grupo nCPAP nos RN } \\
\text { com } \mathrm{PN}<1000 \mathrm{~g}\end{array}$ \\
\hline
\end{tabular}

